\title{
YAGLOM LIMITS CAN DEPEND ON THE STARTING STATE
}

\author{
R. D. FOLEY, ${ }^{*}$ Georgia Institute of Technology \\ D. R. MCDONALD, ${ }^{* *}$ The University of Ottawa
}

\begin{abstract}
We construct a simple example, surely known to Harry Kesten, of an $R$ transient Markov chain on a countable state space $S \cup\{\delta\}$ where $\delta$ is absorbing. The transition matrix $K$ on $S$ is irreducible and strictly substochastic. We determine the Yaglom limit, that is, the limiting conditional behavior given non-absorption. Each starting state $x \in S$ results in a different Yaglom limit. Each Yaglom limit is an $R^{-1}$-invariant quasi-stationary distribution where $R$ is the convergence parameter of $K$. Yaglom limits that depend on the starting state are related to a nontrivial $R^{-1}$-Martin boundary.

Keywords: quasi-stationary; $R$-transient; $\rho$-Martin boundary; Yaglom limit; gambler's ruin; eigenvalue; eigenvector; time reversal; Doob's $h$-transformation; change of measure; invariant measure; harmonic function; substochastic.
\end{abstract}

2010 Mathematics Subject Classification: Primary 60J10

Secondary 60J50

\footnotetext{
* Postal address: Department of Industrial \& Systems Engineering Georgia Institute of Technology

Atlanta, Georgia 30332-0205

U.S.A.

** Postal address: Department of Mathematics and Statistics

The University of Ottawa

Ottawa, Ontario

Canada K1N 6N5
} 
The long run is a misleading guide to current affairs. In the long run we are all dead. Economists set themselves too easy, too useless a task if in tempestuous seasons they can only tell us that when the storm is past the ocean is flat again.

John Maynard Keynes

\section{Introduction}

A gambler is pitted against an infinitely wealthy casino. The gambler enters the casino with $x>0$ dollars. With each play, the gambler either wins a dollar with probability $b$ where $0<b<1 / 2$ or loses a dollar. The gambler continues to play for as long as possible. What can be said about her fortune after many plays given that she still has at least one dollar?

Seneta and Vere-Jones [27] answered this question with the following probability distribution $\pi^{*}$ :

$$
\pi^{*}(y)=\frac{1-\rho}{a} y\left(\sqrt{\frac{b}{a}}\right)^{y-1} \text { for } y=1,2, \ldots
$$

where $a=1-b$ and $\rho=2 \sqrt{a b}$. Let $X_{n}$ be her fortune after $n$ plays. Notice that her fortune alternates between being odd and even. For $n$ large, Seneta and Vere-Jones proved that

$$
\mathbb{P}_{x}\left\{X_{n}=y \mid X_{n} \geq 1\right\} \approx \begin{cases}\frac{\pi^{*}(y)}{\pi^{*}(2 \mathbb{N})} & \text { for } y \text { even and } x+n \text { is even } \\ \frac{\pi^{*}(y)}{\pi^{*}(2 \mathbb{N}-1)} & \text { for } y \text { odd and } x+n \text { is odd }\end{cases}
$$

where $\mathbb{N}:=\{1,2, \ldots\}$ and $\mathbb{P}_{x}$ means that we also condition on $X_{0}=x \in \mathbb{N}$. The probability $\pi^{*}$ assigns to the even and odd natural numbers is denoted by $\pi^{*}(2 \mathbb{N})$ and $\pi^{*}(2 \mathbb{N}-1)$, respectively.

The gambler's ruin problem and the Seneta-Vere-Jones' result are beautiful, but the even/odd periodicity obscures our main point. To remove this distraction, assume the gambler starts with an even number of dollars $2 x$, and consider the Markov chain 
$X_{0}, X_{2}, X_{4}, \ldots$ The transition matrix for this chain restricted to even states that are strictly positive is aperiodic, and

$$
\lim _{n \rightarrow \infty} \mathbb{P}_{2 x}\left\{X_{2 n}=y \mid X_{2 n} \geq 1\right\}=\frac{\pi^{*}(y)}{\pi^{*}(2 \mathbb{N})} \quad \text { for } y \in 2 \mathbb{N} .
$$

Notice that this limiting conditional distribution does not depend on the starting state $2 x$. Whether this holds true in general for irreducible, aperiodic sub-Markov chains is, or was, an open question. There is neither a proof $^{1}$ that if the limiting conditional distributions starting from different states exist, they must be equal, nor an example showing that they might not be. This paper fills that gap. We construct an example where every starting state $x$ leads to a different limiting conditional behavior $\pi_{x}$.

More precisely, consider a sub-Markov chain $X_{0}, X_{1}, \ldots$ on a countably infinite state space $S$. By a sub-Markov chain, we mean that the one-step transition matrix $K$ between states in $S$ is substochastic; that is, $K(x, S):=\sum_{y \in S} K(x, y) \leq 1$. Strictly substochastic means that there is at least one row $x$ such that $K(x, S)<1$. The missing mass can be thought of as representing a transition to an absorbing state $\delta \notin S$. $K$ is irreducible if for any $x, y \in S$, there exists an $n=n(x, y)$ such that $K^{n}(x, y)>0$ where $K^{n}$ is the matrix of $n$-step transition probabilities. $K$ is aperiodic if $d=1$ where $d=\operatorname{gcd}\left\{n>0: K^{n}(x, x)>0\right\}$, which does not depend on $x$ when $K$ is irreducible. If $d>1$, then $K$ is periodic with period $d$.

We construct examples where every starting state $x$ leads to a different limiting conditional distribution $\pi_{x}$ even though the transition matrix is irreducible and aperiodic. That is,

$$
\mathbb{P}_{x}\left\{X_{n}=y \mid X_{n} \in S\right\} \rightarrow \pi_{x}(y) \text { for } y \in S,
$$

but $\pi_{x}$ is different for every $x \in S$.

The paper is organized as follows. The first several subsections of section 2 define some standard terms. In subsection 2.4 we define a limiting condition distributioncalled the Yaglom limit - for the aperiodic case. Our definition is slightly unusual since we explicitly allow the possible dependence upon the starting state by including the

\footnotetext{
${ }^{1}$ This is not exactly true; see the second paragraph of Section 4 of [15].
} 
starting state $x$ in the r.h.s. of (5). We prove several basic results that follow from the existence of a Yaglom limit in the aperiodic case for a fixed starting state $x$. Although Kesten's strong ratio limit property (SRLP) may not hold, we show that a generalized strong ratio limit property (GSRLP) holds when a Yaglom limit exists for each starting state - though each Yaglom limit may be different.

In subsection 2.4 we turn our attention to periodic Yaglom limits where $K$ is periodic with period $d>1$. Many of our examples, e.g., the gambler's ruin problem, are periodic. The definition of a periodic Yaglom limit is given in (11) and again the possible dependence on the starting state is explicit. In addition, the definition includes the sequence of subsets of the state space that the process cycles through. A periodic Yaglom limit requires that $d$ different limits hold. After proving some basic properties that follow from the existence of a periodic Yaglom limit, we establish a series of results that greatly simplify the process of establishing a periodic Yaglom limit.

Section 3 describes a duality between $t$-invariant measures and $t$-harmonic functions related to reversibility. A more general such duality is described in subsection 5.5. Section 4 describes an idea that allows a variety of useful identities to be derived. Section 5 contains a variety of examples including our primary example dubbed the "hub-and-two-spoke example." The hub-and-two-spoke example provides an excellent medium for exploring the connections between Martin boundary theory-particularly, the $\rho$-Martin entrance boundary theory — and Yaglom limits that may depend on the initial state.

Instead of reading section 2 next, we encourage the reader to look at the definitions of a Yaglom limit (5) and a periodic Yaglom limit (11) and then immediately jump to the hub-and-two-spoke example in section 5. Theorem 1 shows that the periodic Yaglom limit of the hub-and-two-spoke example depends on the initial state. This and the other examples will motivate the results in the sections initially skipped. We refer back to the results in the skipped sections as we need them when analyzing the examples. 


\section{Characterizing Yaglom limits}

Throughout, we assume that $K$ is an irreducible, substochastic matrix. Our goal in this section is to characterize the Yaglom limits (2) as quasi-stationary distributions for both the aperiodic and periodic cases.

Let $\zeta$ be the exit time from $S$, also known as the time of absorption. Notice that

$$
\mathbb{P}_{x}\left\{X_{n} \in S\right\}=K^{n}(x, S)=\mathbb{P}_{x}\{\zeta>n\}
$$

and, since $\delta$ is absorbing

$$
\mathbb{P}_{x}\left\{X_{n}=y \mid X_{n} \in S\right\}=\frac{K^{n}(x, y)}{K^{n}(x, S)} \quad \text { for all } y \in S
$$

From Scheffé's Theorem [2, Theorem 16.11], (2) implies convergence in total variation. We frequently appeal to the corollary of Scheffé's Theorem to conclude that

$$
\mathbb{E}_{x}\left[f\left(X_{n}\right) \mid X_{n} \in S\right] \rightarrow \pi_{x} f:=\sum_{y \in S} \pi_{x}(y) f(y) .
$$

whenever $f$ is a bounded function on $S$.

\subsection{Quasi-stationary distributions}

We think of a distribution $\pi$ as a nonnegative row vector with elements $\pi(y)$ for $y \in S$ that sum to $\pi(S) \leq 1$. For $\pi$ to be a quasi-stationary distribution, we need $\pi(S)=1$, and

$$
\mathbb{P}_{\pi}\left\{X_{n}=y \mid X_{n} \in S\right\}=\pi(y) \text { for all } n \geq 0
$$

where $\mathbb{P}_{\pi}$ is the distribution of the chain when $X_{0}$ is given the distribution $\pi$. Irreducibility implies that a quasi-stationary distribution for $K$ must be strictly positive. If $K$ is also strictly substochastic, then $0<\mathbb{P}_{\pi}\left\{X_{1} \in S\right\}<1$. We will use the abbreviation QSD for quasi-stationary distribution, and QSDs for the plural.

\subsection{Invariant and excessive measures and QSDs}

If we set $n=1$ in (3) and multiply both sides by $t:=\mathbb{P}_{\pi}\left\{X_{1} \in S\right\}$, we have $\pi K=t \pi$ where $0<t \leq 1$. If $K$ is strictly substochastic, the factor $t$ shrinks $\pi$ to account for the missing mass. Thus, $\pi$ is a positive left eigenvector for the eigenvalue $t$ and will be called a $t$-invariant QSD. Similarly, a measure $\sigma$ on $S$ is $t$-invariant if $0 \leq \sigma(x)<\infty$ 
for all $x \in S$ and $\sigma K=t \sigma$. If If $0 \leq \sigma(x)<\infty$ for all $x \in S$ and $\sigma K \leq t \sigma$ then $\sigma$ is $t$-excessive. It follows from irreducibility that a non-degenerate $t$-invariant measure with $t>0$ must be strictly positive. Invariant measure means the same as 1 -invariant measure.

We are primarily interested in $\rho$-invariant QSDs where $\rho=1 / R$ and $R$ is the (common) radius of convergence of the generating functions

$$
G_{x, y}(z):=\sum_{n \geq 0} K^{n}(x, y) z^{n}
$$

Seneta [26, Theorem 6.1] has a proof that the radius of convergence of $G_{x, y}(z)$ is the same for every $x, y$ and also that $R<\infty$. In addition, since $K$ is substochastic, $1 \leq R<\infty$. $R$ is called the convergence parameter of $K$, and $\rho=1 / R$ the convergence norm or spectral radius. Either $G_{x, y}(R)<\infty$ for all $x, y$, or $G_{x, y}(R)=\infty$ for all $x, y$. In the former case, $K$ is said to be $R$-transient, and in the latter case, $R$-recurrent. The $R$-recurrent case tends to be far more tractable since there is at most one $\rho$-invariant measure.

$K$ may have $t$-invariant QSDs for $t \neq \rho$. For example, a consequence of Theorem 4.2 in van Doorn and Schrijner [30] is that the gambler's ruin problem described in the Introduction has a unique $t$-invariant QSD for every $t \in[\rho, 1)$. Our reason for focusing on $\rho$-invariant QSDs is that Proposition 1 implies that only $\rho$-invariant QSDs can describe the limiting conditional behavior when the initial distribution is concentrated on a single state. If the initial distribution is allowed to have an infinite support, any $t$-invariant QSD can be a limiting conditional distribution. We give examples where each starting state $x$ results in a different limiting conditional distribution chosen from an infinite family of $\rho$-invariant QSDs.

Seneta and Vere-Jones [27] allow for the possibility that an irreducible, substochastic matrix could have multiple $\rho$-invariant measures. The $\rho$-Martin entrance boundary theory [8] describes the cone of such measures - more on that in subsubsection 5.2.11. Nonetheless, we do not know of any earlier examples of Yaglom limits that depend on the starting state. 


\subsection{Harmonic and superharmonic functions}

Analogous to $t$-invariant and $t$-excessive measures, we have $t$-harmonic and $t$-superharmonic functions. A real-valued function $h$ on $S$ will be $t$-superharmonic if $h \geq 0$ and $K h \leq t h$. To avoid continually writing "nonnegative," we have included nonnegativity as part of the definition of $t$-superharmonic. If in addition, $K h=t h$, then $h$ is $t$-harmonic. Due to irreducibility, a non-degenerate $t$-superharmonic function with $t>0$ must be strictly positive. Harmonic and superharmonic mean the same as 1-harmonic and 1-superharmonic, resp.

\subsection{The aperiodic case}

A proper probability distribution $\pi_{x}$ describes the limiting conditional behavior starting from state $x \in S$ if (2) holds, or equivalently,

$$
\frac{K^{n}(x, y)}{K^{n}(x, S)} \rightarrow \pi_{x}(y) \quad \text { for all } y \in S \text {. }
$$

where $\pi_{x}$ is a proper probability distribution on $S$. We refer to $\pi_{x}$ as being the limiting conditional distribution or as being the Yaglom limit. Our use of the term Yaglom limit is slightly nonstandard since the r.h.s. may depend on the starting state $x$.

Lemma 1. Let $K$ be irreducible, aperiodic, and substochastic. If $\pi_{x}$ is a Yaglom limit as in (5), then the following hold:

$$
\begin{aligned}
& \lim _{n \rightarrow \infty} \frac{K^{n+1}(x, S)}{K^{n}(x, S)}=\rho \\
& \lim _{n \rightarrow \infty} \frac{K^{n+1}(x, y)}{K^{n}(x, y)}=\rho \quad \text { for all } y \in S .
\end{aligned}
$$

Remark 1. Recall that $\zeta$ is the exit time from $S$. If the initial distribution is a $t$ invariant $\mathrm{QSD}$, then $\zeta$ is a geometric random variable, and the conditional probability of remaining in $S$ for one more step is always $t$. Call $\mathbb{P}_{x}\{\zeta>n+1 \mid \zeta>n\}$ the survival probability at age $n$ starting from state $x$. Thus, (6) states that the (one-step) survival probability starting from state $x$ is asymptotically $\rho$. Consequently, if $\pi_{x}$ in (5) is a $t$-invariant QSD, then (6) implies $t=\rho$.

Remark 2. Kesten [18] proves (7) in Lemma 4 under a uniform aperiodicity assumption. He also gives some of the earlier history of this result. Equation (6) can be proven with an argument similar to Kesten's proof of Lemma 4 assuming uniform aperiodicity 
and the existence of a $\rho$-excessive probability measure $\mu$ (that is, $\mu K \leq \rho \mu$ ) [10]. These results are quite general, and do not require a Yaglom limit to hold. If, however, a Yaglom limit holds, then we have the following simple proof.

Proof of Lemma 1. Since $K(y, S)$ is a bounded function in $y$, we can use the corollary to Scheffé's Theorem to show that

$$
\begin{aligned}
\frac{K^{n+1}(x, S)}{K^{n}(x, S)} & =\sum_{y \in S} \frac{K^{n}(x, y)}{K^{n}(x, S)} K(y, S) \\
& \rightarrow \sum_{y \in S} \pi_{x}(y) K(y, S) \\
& =: L \in(0,1] .
\end{aligned}
$$

If we think of the l.h.s. of (5) as being of the form $a_{n} / b_{n}$, we have just argued that $b_{n+1} / b_{n} \rightarrow L$. From (5), we know that $\left(a_{n+1} / a_{n}\right)\left(b_{n} / b_{n+1}\right) \rightarrow 1$; hence, the ratios $a_{n+1} / a_{n}$ must also converge to $L$. To finish the proof, we need only show that $L=\rho$.

Since the root test is stronger than the ratio test, it follows that $a_{n}^{1 / n} \rightarrow L$. If $K$ is aperiodic, Theorem A of [31] implies that $\left[K^{n}(x, y)\right]^{1 / n} \rightarrow \rho$. Since $K^{n}(x, y)=a_{n}$, we know that $L=\rho$.

Proposition 1. Let $K$ be irreducible, aperiodic, and substochastic. If $\pi_{x}$ is a Yaglom limit as in (5), then $\pi_{x}$ is a $\rho$-invariant QSD.

Proof. We essentially repeat the proof of Theorem 3.2 in [27]. Starting from (7),

$$
\begin{aligned}
\rho & =\lim _{n \rightarrow \infty} \frac{\sum_{z} K^{n}(x, z) K(z, y)}{K^{n}(x, y)} \\
& =\lim _{n \rightarrow \infty}\left(\frac{K^{n}(x, S)}{K^{n}(x, y)} \sum_{z} \frac{K^{n}(x, z)}{K^{n}(x, S)} K(z, y)\right) \\
& =\frac{1}{\pi_{x}(y)} \sum_{z} \pi_{x}(z) K(z, y) .
\end{aligned}
$$

In the last step, we used (5) twice, and we again used the corollary of Scheffé's Theorem to interchange the limit and sum since $K(z, y)$ is a bounded function of $z$.

2.4.1. Kesten's strong ratio limit property (SRLP). For a nonnegative matrix $K$-not necessarily substochastic - Kesten [18] defines the strong ratio limit property as the existence of a strictly positive constant $R$, a strictly positive function $h$ on $S$, and a 
strictly positive measure $\pi$ on $S$ such that

$$
\lim _{n \rightarrow \infty} \frac{K^{n+m}(u, v)}{K^{n}(x, y)}=R^{-m} \frac{h(u) \pi(v)}{h(x) \pi(y)} \quad \text { for all states } u, v, x, y \text { and all } m \in \mathbb{Z} .
$$

Our example in subsection 5.2 shows that an irreducible, aperiodic substochastic matrix may not have Kesten's strong ratio limit property. We are able to prove a different property that our example does possess and will prove useful. Let us say that a nonnegative matrix $K$ has the generalized strong ratio limit property (GSRLP) if there exists a strictly positive constant $R$, a strictly positive function $h$, and $\rho$-invariant QSDs $\pi_{x}$ on $S$ for every $x \in S$ such that

$$
\lim _{n \rightarrow \infty} \frac{K^{n+m}(u, v)}{K^{n}(x, y)}=R^{-m} \frac{h(u) \pi_{u}(v)}{h(x) \pi_{x}(y)} \quad \text { for all states } u, v, x, y \text { and all } m \in \mathbb{Z} .
$$

Much of the remainder of this subsection is closely related to the proof of Kesten's Theorem 2 [18].

If $K$ is substochastic, irreducible and (5) holds for a fixed state $x_{0}$, then $K^{n}(y, S) / K^{n}\left(x_{0}, S\right)$ is a bounded sequence in $n$ for each $y \in S$. To see this, choose $m$ so that $K^{m}\left(x_{0}, y\right)>0$, and then we have

$$
K^{m}\left(x_{0}, y\right) \frac{K^{n}(y, S)}{K^{n}\left(x_{0}, S\right)} \leq \frac{K^{n+m}\left(x_{0}, S\right)}{K^{n}\left(x_{0}, S\right)} \rightarrow \rho^{m} \quad \text { using (6). }
$$

Since $K^{n}(y, S) / K^{n}\left(x_{0}, S\right)$ is bounded, we can choose a convergent subsequence $\mathcal{N}(y)$ for each $y$, and define the subsequential limit

$$
\hat{h}(y):=\lim _{\substack{n \rightarrow \infty \\ n \in \mathcal{N}(y)}} \frac{K^{n}(y, S)}{K^{n}\left(x_{0}, S\right)} \quad \text { for all } y \in S
$$

If (9) holds for all subsequences, that is, if

$$
\hat{h}(y)=\lim _{n \rightarrow \infty} \frac{K^{n}(y, S)}{K^{n}\left(x_{0}, S\right)} \quad \text { for all } y \in S
$$

then

$$
\frac{\hat{h}(y)}{\hat{h}(z)}=\lim _{n \rightarrow \infty} \frac{K^{n}(y, S)}{K^{n}(z, S)} \quad \text { for all } y, z \in S
$$

and $\hat{h}\left(x_{0}\right)=1$. When (10) holds, we will say that the Jacka-Roberts condition [15, Lemma 2.3] holds. 
Proposition 2. Let $K$ be irreducible, aperiodic, and substochastic. If (5) holds for every $x \in S$ and the Jacka-Roberts condition (10) holds, then the generalized ratio limit property (8) holds, and $\hat{h}$ is $\rho$-superharmonic. If in addition the support of $K(x, \cdot)$ is finite for every $x \in S$, then $\hat{h}$ is $\rho$-harmonic.

Remark 3. The periodic example in subsection 5.3 can be modified to show that $\hat{h}$ may not be $\rho$-harmonic if the finite support assumption does not hold. The JackaRoberts condition (10) plays a key role; throughout we use $\hat{h}$ to designate this survival ratio.

Proof of Proposition 2. First, we argue that $\hat{h}$ is strictly positive. We know that $\hat{h}\left(x_{0}\right)=1$. Choose $m$ such that $K^{m}\left(x, x_{0}\right)>0$. Now,

$$
\begin{array}{rlrl}
K^{m+n}(x, S) & =\sum_{z} K^{m}(x, z) K^{n}(z, S) & \\
\frac{K^{m+n}(x, S)}{K^{m+n}\left(x_{0}, S\right)} & =\sum_{z} K^{m}(x, z) \frac{K^{n}(z, S)}{K^{n}\left(x_{0}, S\right)} \frac{K^{n}\left(x_{0}, S\right)}{K^{m+n}\left(x_{0}, S\right)} & & \text { let } n \rightarrow \infty, \\
\hat{h}(x) & \geq \sum_{z} K^{m}(x, z) \hat{h}(z) R^{m} & \text { from Fatou's lemma } \\
& \geq K^{m}\left(x, x_{0}\right) \hat{h}\left(x_{0}\right) R^{m} & \\
& >0 &
\end{array}
$$

since we already know that $R=\rho^{-1}>0$.

Next, to see that (8) holds,

$$
\frac{K^{m+n}(u, v)}{K^{n}(x, y)}=\frac{K^{m+n}(u, v)}{K^{n}(u, v)} \frac{K^{n}(u, v)}{K^{n}(u, S)} \frac{K^{n}(u, S)}{K^{n}(x, S)} \frac{K^{n}(x, S)}{K^{n}(x, y)} \rightarrow \rho^{m} \frac{\pi_{u}(v) \hat{h}(u)}{\pi_{x}(y) \hat{h}(x)}
$$

where Proposition 1 guarantees that $\pi_{x}$ is a $\rho$-invariant QSD for every $x \in S$.

To show that $\hat{h}$ is $\rho$-harmonic when the support of $K(x, \cdot)$ is finite, return to the argument at the beginning of this proof, and set $m=1$. Instead of using Fatou's lemma, use the finite support to replace the first inequality with equality, which shows that $\hat{h}$ is $\rho$-harmonic.

Even if $\hat{h}$ is known to be $\rho$-harmonic, it can still be difficult to determine $\hat{h}$ since $K$ may have other $\rho$-harmonic functions as our hub-and-two-spoke example illustrates; see (42). Often, Kesten's Theorem 1 [18] can help since it gives conditions guaranteeing a unique, up to multiplicative constants, $\rho$-harmonic function. 
One vexing open question is that we do not know whether the existence of a Yaglom limit starting from a particular state $x$ implies the existence of Yaglom limits starting from other states.

\subsection{Periodic Yaglom limits \& QSDs}

Fix the starting state $x \in S$. If the period $d>1$, then we can partition $S$ into $d$ classes labeled $S_{0}, \ldots, S_{d-1}$ so that $y \in S_{k}$ iff $K^{n d+k}(x, y)>0$ for $n$ sufficiently large. Hence, $x \in S_{0}$, and $\left\{X_{n d+k} \in S_{k}\right\}=\left\{X_{n d+k} \in S\right\}$. For brevity, let $S_{k+j}:=$ $S_{k+j}(\bmod d)$. We will say that $K$ has a periodic Yaglom limit starting from $x$ if for all $k \in\{0, \ldots, d-1\}$ and all $y \in S_{k}$,

$$
\lim _{n \rightarrow \infty} \mathbb{P}_{x}\left\{X_{n d+k}=y \mid X_{n d+k} \in S\right\}=\frac{\pi_{x}(y)}{\pi_{x}\left(S_{k}\right)}
$$

where $\pi_{x}$ is a probability measure on $S$ with $\pi_{x}(S)=1$. We will show $\pi_{x}$ must be a $\rho$-invariant QSD. Unlike when $K$ is stochastic, $\pi_{x}\left(S_{k}\right)$ may take values other than $1 / d$.

Our starting point for the periodic case is slightly different than (2). Instead, we assume that there exists a $k$ such that

$$
\lim _{n \rightarrow \infty} \mathbb{P}_{x}\left\{X_{n d+k}=y \mid X_{n d+k} \in S\right\}=\pi_{x}^{k}(y) \quad \text { for all } y \in S_{k},
$$

or equivalently

$$
\lim _{n \rightarrow \infty} \frac{K^{n d+k}(x, y)}{K^{n d+k}(x, S)}=\pi_{x}^{k}(y) \quad \text { for all } y \in S_{k}
$$

where $\pi_{x}^{k}$ is a probability measure on $S$ with $\pi_{x}^{k}\left(S_{k}\right)=1$. If (13) holds for some $k$, then Proposition 3 shows that we have a periodic Yaglom limit starting from $x$; that is, (11) holds for all $k$, and $\pi_{x}$ is a $\rho$-invariant QSD.

If a periodic Yaglom limit holds for some $x \in S_{0}$, we have not been able to prove that a periodic Yaglom limit holds starting from some other state in $S_{0}$. However, if a periodic Yaglom limit holds for every state in $S_{0}$, then Proposition 4 shows that a periodic Yaglom limit holds starting from any state in $S$.

Lemma 2. Let $K$ be irreducible, substochastic, and periodic with period $d>1$. If (13) 
holds for some $k \in\{0, \ldots, d-1\}$, then

$$
\begin{aligned}
& \lim _{n \rightarrow \infty} \frac{K^{(n+1) d+k}\left(x, S_{k}\right)}{K^{n d+k}\left(x, S_{k}\right)}=\rho^{d} \\
& \lim _{n \rightarrow \infty} \frac{K^{(n+1) d+k}(x, y)}{K^{n d+k}(x, y)}=\rho^{d} \quad \text { for } y \in S_{k} .
\end{aligned}
$$

Proof. Using the corollary to Scheffés Theorem,

$$
\begin{aligned}
\frac{K^{(n+1) d+k}(x, S)}{K^{n d+k}(x, S)} & =\sum_{y \in S} \frac{K^{n d+k}(x, y)}{K^{n d+k}(x, S)} K^{d}(y, S) \\
& \rightarrow \sum_{y \in S} \pi_{x}^{k}(y) K^{d}(y, S) \\
& =: L \in(0,1] .
\end{aligned}
$$

As in the proof of Lemma 1, (13) implies that for any $y \in S_{k}$,

$$
\frac{K^{(n+1) d+k}(x, S)}{K^{n d+k}(x, S)} \sim \frac{K^{(n+1) d+k}(x, y)}{K^{n d+k}(x, y)} \rightarrow L .
$$

Since the root test is stronger ${ }^{2}$ than the ratio test, $\left[K^{n d+k}(x, y)\right]^{1 /(n d)} \rightarrow L^{1 / d}$, but Theorem A [31] implies that for any $y \in S_{k},\left[K^{n d+k}(x, y)\right]^{1 /(n d+k)} \rightarrow \rho$. Hence, $L=\rho^{d}$.

Proposition 3. Let $K$ be irreducible, substochastic, and periodic with period $d>1$. Recall that $x$ is a fixed state in $S_{0}$. Suppose that (13) holds for some $k \in\{0,1, \ldots, d-1\}$. Then (13) holds for all $k \in\{0,1, \ldots, d-1\}$. Moreover, there is a $\rho$-invariant $Q S D \pi_{x}$ such that $\pi_{x}^{k}(y)=\pi_{x}(y) / \pi_{x}\left(S_{k}\right)$ for $y \in S_{k}$ for each $k \in\{0,1, \ldots, d-1\}$. Hence,

$$
\lim _{n \rightarrow \infty} \mathbb{P}_{x}\left\{X_{n d+k}=y \mid X_{n d+k} \in S\right\}=\frac{\pi_{x}(y)}{\pi_{x}\left(S_{k}\right)} \quad \text { for all } k \text { and all } y \in S_{k} .
$$

In addition, the $\pi_{x}^{k}(y)$ in (13) can be iteratively computed by using

$$
\pi_{x}^{k+1 \quad(\bmod d)}(y)=\frac{1}{\rho_{k}(x)} \sum_{z \in S_{k}} \pi_{x}^{k}(z) K(z, y)
$$

where the probability of surviving for one more step given the current distribution is $\pi_{x}^{k}$ is given by

$$
\rho_{k}(x)=\sum_{z \in S_{k}} \pi_{x}^{k}(z) K(z, S)
$$

\footnotetext{
${ }^{2}$ See Clark [4, 11.5.3 and Corollary 255] or [5, 2.5] for a discussion.
} 
Furthermore, the $\rho$-invariant $Q S D \pi_{x}$ can be constructed as

$$
\pi_{x}=\sum_{k=0}^{d-1} c_{k} \pi_{x}^{k}
$$

where

$$
\begin{aligned}
& c_{0}=\frac{1}{1+\frac{\rho_{0}(x)}{\rho}+\frac{\rho_{0}(x) \rho_{1}(x)}{\rho^{2}}+\cdots+\frac{\rho_{0}(x) \rho_{1}(x) \ldots \rho_{d-2}(x)}{\rho^{d-1}}} \\
& c_{k}=c_{0} \frac{\rho_{0}(x) \ldots \rho_{k-1}(x)}{\rho^{k}} \quad \text { for } k \in\{1,2, \ldots, d-1\}
\end{aligned}
$$

Remark 4. Lemma 11 of Ferrari and Rolla [9] and Proposition 3 are closely related. Equations (20) and that $c_{k} \rho_{k} / \rho=c_{k+1}$ are in their Lemma 11.

Remark 5. In the following special case, $\rho_{k}(x)$ is easy to compute. Let $\Delta:=\{x \in S$ : $K(x, S)<1\}$ be the set of possible exit states. If $\Delta \subset S_{j}$ for some $j$, then

$$
\rho_{k}(x)= \begin{cases}\rho^{d} & \text { for } k=j \\ 1 & \text { for } k \neq j\end{cases}
$$

which follows from (20) below.

Proof. Recall that $\zeta$ is the exit time from $S$. Let $s_{n}:=s_{n}(x):=\mathbb{P}_{x}\{\zeta>n+1 \mid \zeta>n\}$ for $n \in \mathbb{N}_{0}:=\{0\} \cup \mathbb{N}$. Clearly, $s_{n}>0$, and so is $\inf _{n} s_{n}$ since (14) implies that $\liminf _{n} s_{n} \geq \rho^{d}>0$.

We now show that $s_{n d+k} \rightarrow \rho_{k}$ where $\rho_{k}:=\rho_{k}(x)=\sum_{z \in S_{k}} \pi_{x}^{k}(z) K\left(z, S_{k+1}\right)$.

$$
\begin{aligned}
1-\frac{\rho_{k}}{s_{n d+k}} & =\frac{\sum_{z \in S_{k}} K^{n d+k}(x, z) K\left(z, S_{k+1}\right)}{K^{n d+k}\left(x, S_{k}\right) s_{n d+k}}-\frac{\sum_{z \in S_{k}} \pi_{x}^{k}(z) K\left(z, S_{k+1}\right)}{s_{n d+k}} \\
& \leq \sum_{z \in S_{k}}\left|\frac{K^{n d+k}(x, z)}{K^{n d+k}\left(x, S_{k}\right)}-\pi_{x}^{k}(z)\right| \frac{K\left(z, S_{k+1}\right)}{s_{n d+k}} \\
& \leq \sum_{z \in S_{k}}\left|\frac{K^{n d+k}(x, z)}{K^{n d+k}\left(x, S_{k}\right)}-\pi_{x}^{k}(z)\right| \frac{1}{\inf _{n} s_{n d+k}} \\
& \rightarrow 0 .
\end{aligned}
$$

where we used (13).

For brevity, write $k+1$ for $k+1(\bmod d)$. The next step is to establish that (13) 
holds when $k$ is replaced by $k+1$ and to relate $\pi_{x}^{k+1}$ with $\pi_{x}^{k}$. For $y \in S_{k+1}$,

$$
\begin{aligned}
\frac{K^{n d+k+1}(x, y)}{K^{n d+k+1}\left(x, S_{k+1}\right)} & =\sum_{z \in S_{k}} \frac{K^{n d+k}(x, z)}{K^{n d+k}\left(x, S_{k}\right)} K(z, y) \frac{1}{s_{n d+k}} \\
& \rightarrow \sum_{z \in S_{k}} \pi_{x}^{k}(z) K(z, y) \frac{1}{\rho_{k}} \\
& =: \pi_{x}^{k+1}(y) .
\end{aligned}
$$

Thus, $\pi_{x}^{k+1}$ is a probability measure on $S$ with $\pi_{x}^{k+1}\left(S_{k+1}\right)=1$, as can be seen by summing over $y \in S_{k+1}$. Thus, (15) holds.

Now that we have established that (13) holds when $k$ is replaced by $k+1$, we can repeat the above arguments $d-2$ additional times establishing that the above expressions in this proof hold for all $k \in\{0, \ldots, d-1\}$.

Next, since

$$
\begin{aligned}
\frac{K^{(n+1) d+k}\left(x, S_{k}\right)}{K^{n d+k}\left(x, S_{k}\right)} & =\prod_{j=0}^{d-1} s_{n d+k+j}, \\
\rho^{d} & =\rho_{0}(x) \rho_{1}(x) \ldots \rho_{d-1}(x) \quad \text { as } n \rightarrow \infty
\end{aligned}
$$

where we used (14).

Though the notation suppresses the dependency, $\rho_{k}, s_{n d+k}$ and the forthcoming $c_{0}, \ldots, c_{d-1}$ all depend on the initial state being $x$. Construct $c_{0}, \ldots, c_{d-1}$ as in (17) and (18). The constants are nonnegative, and $c_{0}+\cdots+c_{d-1}=1$. Define $\pi_{x}$ as in (16). Since $\pi_{x}\left(S_{k}\right)=c_{k}, \pi_{x}$ is a probability measure on $S$, and if $y \in S_{k}$, then $\pi_{x}^{k}(y)=\pi_{x}(y) / \pi_{x}\left(S_{k}\right)$ for all $k$.

The last step is to show that $\pi_{x}$ is $\rho$-invariant. We will use the fact that $c_{k} \rho_{k} / \rho=$ $c_{k+1}$ and that from (19),

$$
\rho_{k} \pi_{x}^{k+1}(y)=\sum_{z \in S_{k}} \pi_{x}^{k}(z) K(z, y)
$$

Let $y \in S$. Since $y \in S$, there exists some $k=k(y)$ such that $y \in S_{k+1}$. Since

$$
\begin{aligned}
\sum_{z \in S} \pi_{x}(z) K(z, y) & =\sum_{z \in S_{k}} c_{k} \pi_{x}^{k}(z) K(z, y) \\
& =c_{k} \rho_{k} \pi_{x}^{k+1}(y) \\
& =\rho c_{k+1} \pi_{x}^{k+1}(y) \\
& =\rho \pi_{x}(y),
\end{aligned}
$$


$\pi_{x}$ is a $\rho$-invariant QSD.

If we assume that a periodic Yaglom limit exists starting from every state in one class, say $S_{0}$, then we can show that under certain conditions a periodic Yaglom limit will exist starting from every state in $S$. Since we will be assuming that a periodic Yaglom limit exists starting from any state in $S_{0}$, we drop the assumption that $x$ is a fixed starting state in $S_{0}$, which means that we need to be careful about quantities that depend on the starting state. However, we will still need a fixed state $x_{0}$ in $S_{0}$ as a reference point for the following definition. If the following limit exists, which is similar to (10) except with $n d$ instead of $n$, then define

$$
\hat{h}_{0}(y):=\lim _{n \rightarrow \infty} \frac{K^{n d}\left(y, S_{0}\right)}{K^{n d}\left(x_{0}, S_{0}\right)} \quad \text { for all } y \in S_{0} .
$$

If (21) holds, then it follows that

$$
\frac{\hat{h}_{0}(y)}{\hat{h}_{0}(x)}=\lim _{n \rightarrow \infty} \frac{K^{n d}\left(y, S_{0}\right)}{K^{n d}\left(x, S_{0}\right)} \quad \text { for all } x, y \in S_{0} .
$$

Equation 21 is the Jacka-Roberts condition (10) for the process watched on $S_{0}$.

In the following proposition when the starting state is in a class other than $S_{0}$, do not relabel the classes.

Proposition 4. Let $K$ be irreducible, substochastic, and periodic with period $d>1$. If (12) holds for all $x \in S_{0},(21)$ holds, and the support of $K(u, \cdot)$ is finite for all $u \in S$, then there is a periodic Yaglom limit starting from any state in S. More precisely, if $u \in S_{j}$ where $j \in\{1, \ldots, d-1\}$, then

$$
\frac{K^{n d+k-j}(u, y)}{K^{n d+k-j}\left(u, S_{k}\right)} \rightarrow \frac{\pi_{u}(y)}{\pi_{u}\left(S_{k}\right)} \quad \text { for all } k \text { and all } y \in S_{k}
$$

where $\pi_{u}$ is a $\rho$-invariant QSD. In particular, for $k=0$, the r.h.s. of (22) is given by

$$
\begin{aligned}
\pi_{u}^{-j}(y) & :=\sum_{x \in S_{0}} w_{u, x} \pi_{x}^{0}(y) & \text { for } y \in S_{0}, \\
w_{u, x} & :=\frac{K^{d-j}(u, x) \hat{h}_{0}(x)}{\sum_{z \in S_{0}} K^{d-j}(u, z) \hat{h}_{0}(z)} &
\end{aligned}
$$

where the weights $w_{u, x}$ are nonnegative and sum to one. In addition,

$$
\frac{K^{n d+d-j}\left(u, S_{0}\right)}{K^{n d+d-j}\left(v, S_{0}\right)} \rightarrow \frac{\sum_{x \in S_{0}} K^{d-j}(u, x) \hat{h}_{0}(x)}{\sum_{z \in S_{0}} K^{d-j}(v, z) \hat{h}_{0}(z)}
$$


Remark 6. The terms involving $\hat{h}_{0}(\cdot)$ account for the relative likelihood of a long remaining lifetime, which might vary over the states in $S_{0}$. If the hypotheses of Prop. 6 hold, then the denominator in (24) simplifies to $\rho^{d-j} \hat{h}(u)$ where $\hat{h}(u)$ is defined just prior to Prop. 6.

The example in subsection 5.3 shows that $\hat{h}$ may not be $\rho$-harmonic if the finite support assumption does not hold.

Proof. It suffices to establish that (22) holds for any $j \in\{1, \ldots, d-1\}$ but only one $k$ since Prop. 3 can then be used to show that (22) holds for all $k$ and that $\pi_{u}$ is a $\rho$-invariant QSD. We will show that it holds for $k=0$ by letting $k=d$ in the l.h.s. of (22) and showing that it converges to the r.h.s. of (23). At which point, everything follows from Prop. 3 except (25).

Consider the numerator of the l.h.s. of (22) with $k=d$ divided by $K^{n d}\left(x_{0}, S_{0}\right)$. That is, for $y \in S_{0}$,

$$
\begin{aligned}
\frac{K^{(n+1) d-j}(u, y)}{K^{n d}\left(x_{0}, S_{0}\right)} & =\sum_{x \in S_{0}} K^{d-j}(u, x) \frac{K^{n d}(x, y)}{K^{n d}\left(x, S_{0}\right)} \frac{K^{n d}\left(x, S_{0}\right)}{K^{n d}\left(x_{0}, S_{0}\right)} \\
& \rightarrow \sum_{x \in S_{0}} K^{d-j}(u, x) \pi_{x}^{0}(y) \hat{h}_{0}(x)
\end{aligned}
$$

where we used the finite support of $K^{d-j}(u, \cdot)$ to justify interchanging the limit and summation and then used (21) and (12).

Now, consider the denominator of the l.h.s. of (22) also with $k=d$ and also divided by $K^{n d}\left(x_{0}, S_{0}\right)$. That is,

$$
\begin{aligned}
\frac{K^{(n+1) d-j}\left(u, S_{0}\right)}{K^{n d}\left(x_{0}, S_{0}\right)} & =\sum_{x \in S_{0}} K^{d-j}(u, x) \frac{K^{n d}\left(x, S_{0}\right)}{K^{n d}\left(x_{0}, S_{0}\right)} \\
& \rightarrow \sum_{x \in S_{0}} K^{d-j}(u, x) \hat{h}_{0}(x)
\end{aligned}
$$

where we again used the finite support of $K^{d-j}(u, \cdot)$ to justify interchanging the limit and summation and then used (21).

Combining the above, the l.h.s. of (22) with $k=d$ has a limit

$$
\begin{aligned}
\pi_{u}^{-j}(y) & :=\frac{\sum_{x \in S_{0}} K^{d-j}(u, x) \pi_{x}^{0}(y) \hat{h}_{0}(x)}{\sum_{z \in S_{0}} K^{d-j}(u, z) \hat{h}_{0}(z)} & & \text { for } y \in S_{0} \\
& =\sum_{x \in S_{0}} w(u, x) \pi_{x}^{0}(y) & & \text { for } y \in S_{0}
\end{aligned}
$$


where $w(u, x)$ is defined in (24). Clearly, $w(u, x)>0$ and $\sum_{x \in S_{0}} w(u, x)=1$. The limit $\pi_{u}^{-j}(y)$ is also nonnegative and $\sum_{y \in S_{0}} \pi_{u}^{-j}(y)=1$. Thus, after a temporary relabelling of $S_{0}, \ldots, S_{d-1}$, we can use Prop. 3 to establish (22) for all $k$.

Lastly, to establish (25), just use the result related to the denominator of the l.h.s. of (22) starting from $u$ and the analogous result starting from $v$.

Recall that in the proof of Prop. 3, we showed that $\mathbb{P}_{x}\{\zeta>n d+k+1 \mid \zeta>n d+k\} \rightarrow$ $\rho_{k}(x)$ where $x \in S_{0}$, which gives the asymptotic probability of surviving one more step given that the process is currently in $S_{k}$ and started in state $x$. Part of the hypothesis of the forthcoming Prop. 6 will be that each of the functions $\rho_{0}(\cdot), \ldots, \rho_{d-1}(\cdot)$ is a constant; that is,

$$
\rho_{k}(x)=\rho_{k}(y) \quad \text { for all } k \text {, and all } x, y \in S_{0} .
$$

We will see an example where (26) holds even though $\pi_{x} \neq \pi_{y}$ whenever $x \neq y$. Remark 5 gave one sufficient condition for - something stronger than-(26) to hold. A second sufficient condition for (26) is given in the following proposition.

Proposition 5. Let $K$ be irreducible, substochastic, and periodic with period $d>1$. If (12) holds for all $x \in S_{0}$ and if the following limits exist

$$
\lim _{n \rightarrow \infty} \frac{K^{n}(x, S)}{K^{n}\left(x_{0}, S\right)} \quad \text { for all } x \in S_{0},
$$

then (26) holds for all $k \in\{0, \ldots, d-1\}$.

Proof. From the proof of Prop. 3,

$$
\frac{K^{n d+k+1}\left(x, S_{k+1}\right)}{K^{n d+k}\left(x, S_{k}\right)} \rightarrow \rho_{k}(x) \quad \text { for } x \in S_{0}
$$

where we used (12) and the corollary to Scheffé's Theorem in the last step. Consequently, for $x, y \in S_{0}$,

$$
\left(\frac{K^{n d+k+1}\left(x, S_{k+1}\right)}{K^{n d+k}\left(x, S_{k}\right)}\right)\left(\frac{K^{n d+k}\left(y, S_{k}\right)}{K^{n d+k+1}\left(y, S_{k+1}\right)}\right) \rightarrow \frac{\rho_{k}(x)}{\rho_{k}(y)} .
$$


On the other hand, the above limit could also be evaluated using (27). If the limits in (27) hold, then the limits must be equal to $\hat{h}_{0}(x)$ where $\hat{h}_{0}$ was defined in (21) Consequently,

$$
\left(\frac{K^{n d+k+1}\left(x, S_{k+1}\right)}{K^{n d+k+1}\left(y, S_{k+1}\right)}\right)\left(\frac{K^{n d+k}\left(y, S_{k}\right)}{K^{n d+k}\left(x, S_{k}\right)}\right) \rightarrow \frac{\hat{h}_{0}(x)}{\hat{h}_{0}(y)} \cdot \frac{\hat{h}_{0}(y)}{\hat{h}_{0}(x)}=1,
$$

which means that $\rho_{k}(x)=\rho_{k}(y)$ for all $k$.

If (21) holds, define $\hat{h}(u)$ for $u \in S$ as follows: if $u \in S_{j}$, then

$$
\hat{h}(u):=\rho^{j-d} \sum_{x \in S_{0}} K^{d-j}(u, x) \hat{h}_{0}(x)
$$

where $j \in\{0, \ldots, d-1\}$. Similar to the aperiodic case, $\hat{h}$ is some sort of measure of the likelihood of surviving for a long time relative to a fixed state $x_{0}$. The definition of $\hat{h}$ seems to be the correct way to extend $\hat{h}_{0}$ from a function on $S_{0}$ to a function on $S$ so that (28) holds and so that $\hat{h}$ has a chance of being $\rho$-harmonic. The following proposition gives conditions for $\hat{h}$ to be $\rho$-harmonic. Although there may be many other $\rho$-harmonic functions, $\hat{h}$ plays an important role in the large deviation behavior.

Proposition 6. Let $K$ be irreducible, substochastic, and periodic with period $d>1$. If (12) holds for all $x \in S_{0}$, (21) and (26) hold, and the support of $K(u, \cdot)$ is finite for all $u \in S$, then $\hat{h}$ is $\rho$-harmonic. If in addition $u$ and $v$ are in the same class $S_{j}$, then

$$
\frac{\hat{h}(v)}{\hat{h}(u)}=\lim _{n \rightarrow \infty} \frac{K^{n}(v, S)}{K^{n}(u, S)}
$$

Remark 7. The example in subsection 5.3 shows that $\hat{h}$ may not be $\rho$-harmonic without the finite support assumption.

Proof. First, we show that $\hat{h}$ is $\rho$-harmonic. Fix $j \in\{0, \ldots, d-1\}$. Let $u \in S_{j}$ and $v \in S_{j+1}$ (where by convention $S_{d}$ means $S_{0}$ ).

$$
\begin{aligned}
\sum_{v} K(u, v) \hat{h}(v) & =\sum_{v} K(u, v) \rho^{j+1-d} \sum_{x \in S_{0}} K^{d-(j+1)}(v, x) \hat{h}_{0}(x) \\
& =\rho \rho^{j-d} \sum_{x \in S_{0}} K^{d-j}(u, x) \hat{h}_{0}(x) \\
& =\rho \hat{h}(u) .
\end{aligned}
$$


Next, we prove the ratio limit result. Let $u \in S_{j}$ for fixed $j \in\{0, \ldots, d-1\}$ From (26), $\rho_{k}(x)$ does not depend on $x$, so we shorten it to $\rho_{k}$. Fix $i \in\{1, \ldots, d\}$.

$$
\begin{aligned}
K^{d-j+m d+i}(u, S) & =\sum_{x, y \in S_{0}} K^{d-j}(u, x) K^{m d}(x, y) K^{i}\left(y, S_{i}\right) \\
\frac{K^{d-j+m d+i}(u, S)}{K^{m d}\left(x_{0}, S_{0}\right)} & =\sum_{x, y \in S_{0}} K^{d-j}(u, x) \frac{K^{m d}(x, y)}{K^{m d}\left(x, S_{0}\right)} K^{i}\left(y, S_{i}\right) \frac{K^{m d}\left(x, S_{0}\right)}{K^{m d}\left(x_{0}, S_{0}\right)} \\
& \rightarrow \sum_{x, y \in S_{0}} K^{d-j}(u, x) \pi_{x}^{0}(y) \rho_{0} \ldots \rho_{i-1} \hat{h}_{0}(x) \quad \text { as } m \rightarrow \infty \\
& =\rho_{0} \ldots \rho_{i-1} \sum_{x \in S_{0}} K^{d-j}(u, x) \hat{h}_{0}(x) \\
& =\rho_{0} \ldots \rho_{i-1} \rho^{d-j} \hat{h}(u)
\end{aligned}
$$

where we justify interchanging the limit and sum in the next paragraph. In the above, we used the identity $\sum_{y \in S_{0}} \pi_{x}^{0}(y) K^{j}\left(y, S_{j}\right)=\rho_{0}(x) \ldots \rho_{j-1}(x)$. Also, since the $\rho_{k}$ 's do not depend on $x$, they could be factored outside the summation. Consequently, if $v$ is also in $S_{i}$, then

$$
\frac{K^{d-j+m d+i}(u, S)}{K^{d-j+m d+i}(v, S)} \rightarrow \frac{\hat{h}(u)}{\hat{h}(v)} \quad \text { as } m \rightarrow \infty .
$$

Since this limit does not depend on $i$ and $j$ as long as $u$ and $v$ are in the same class, (28) holds.

Since there are only a finite number of states $x$ accessible from $u$, it suffices to show that we can interchange the limit and the sum over $y \in S_{0}$. Since $K^{m d}(x, y) / K^{m d}\left(x_{0}, S_{0}\right)$ converges to $\pi_{x}^{0}(y) \rho_{0} \ldots \rho_{i-1} \hat{h}_{0}(x)$, which is summable over $y \in S_{0}$, and since $K^{i}\left(y, S_{i}\right)$ is bounded, we can again use the corollary to Scheffe's theorem to justify interchanging the limit and sum.

\section{Duality and reversibility}

In some situations, a duality exists between $t$-invariant measures and $t$-harmonic functions. The example in subsection 5.3 is a situation where they cannot be linked since there is a $\rho$-invariant measure, but no $\rho$-harmonic function. We now describe a situation where such a duality arises and is related to a kind of reversibility for substochastic matrices; there will be additional duality discussion without reversibility in subsection 5.5. 
First, we need several definitions. Given a positive $t$-harmonic function $h$ on $S$, Doob's $h$-transform of $K$, sometimes called the twisted kernel, is given by

$$
\tilde{K}(x, y)=\frac{K(x, y) h(y)}{\operatorname{th}(x)} .
$$

Similarly, given a positive $t$-invariant measure $\sigma$ on $S$, the time reversal with respect to $\sigma$ is

$$
\overleftarrow{K}(x, y)=\frac{\sigma(y) K(y, x)}{t \sigma(x)}
$$

Both $\overleftarrow{K}$ and $\tilde{K}$ are stochastic matrices on $S$. There may be many different $t$-harmonic functions, and many different $t$-invariant measures. If $\tilde{K}=\overleftarrow{K}$ and the same eigenvalue $t$ was used in constructing both, then the $h$ used in constructing $\tilde{K}$ and the $\sigma$ used in constructing $\overleftarrow{K}$ are duals.

We call $K$ reversible if the Kolmogorov criterion holds; that is, if

$$
K\left(x_{0}, x_{1}\right) K\left(x_{1}, x_{2}\right) \cdots K\left(x_{n-1}, x_{n}\right)=K\left(x_{n}, x_{n-1}\right) K\left(x_{n-1}, x_{n-2}\right) \cdots K\left(x_{1}, x_{0}\right)
$$

for any sequence of states $x_{0}, x_{1}, \ldots, x_{n}$ with $x_{0}=x_{n}$. The following results are part of Theorems 4.1 in Pollett [23] and in Pollett[24].

Proposition 7. (Pollett[24].) Let $K$ be irreducible and substochastic on $S . K$ is reversible iff there exists a positive measure $\gamma$ on $S$ such that

$$
\gamma(x) K(x, y)=\gamma(y) K(y, x) \quad \text { for all } x, y \in S .
$$

If $\tilde{K}=\overleftarrow{K}$ where both were computed using the same eigenvalue $t$, then $K$ is reversible. If $K$ is reversible, $\sigma$ is t-invariant, $h$ is t-harmonic, and $\sigma(x)=h(x) / \gamma(x)$, then $\tilde{K}=\overleftarrow{K}$. If $K$ is reversible and $\sigma$ is a t-invariant measure, then $h(x):=\sigma(x) / \gamma(x)$ for $x \in S$ defines the dual t-harmonic function. Similarly, if $K$ is reversible and $h$ is a t-harmonic function, then $\sigma(x):=h(x) \gamma(x)$ for $x \in S$ defines the dual t-invariant measure.

Remark 8. In the stochastic case, a constant function $h$ is 1-harmonic so $\tilde{K}=$ $\overleftarrow{K}$ becomes the familiar $K=\overleftarrow{K}$, though without the assumption of stationarity. For example, consider a simple random walk on the integers that moves right with probability $0<p<1 / 2$. Birth-death processes are reversible since they satisfy 
Kolmogorov's criterion, and for this process with $h$ being a column vector of ones, we have $\gamma(x)=\sigma(x)=(p /(1-p))^{x}$. For this pair, $\tilde{K}=\overleftarrow{K}$, and the associated Markov chains drift to negative infinity. There is another 1-harmonic function: $((1-p) / p)^{x}$. The corresponding 1-invariant measure must be obtained by multiplying by $\gamma(x)$ giving $(\ldots, 1,1,1, \ldots)$. Both the time reversal and the twisted process for this pair drift to plus infinity. Notice that a single reversibility measure $\gamma$ works for all eigenvalues $t$.

Proof. The following parts of the argument related to Kolmogorov's criterion is quite similar to the argument in the proof of Theorem 1.7 in [16] though without stationarity and for a substochastic matrix. For any path $x=\left(x_{0}, x_{1}, \ldots, x_{n}\right)$, let $\kappa(x):=K\left(x_{0}, x_{1}\right) K\left(x_{1}, x_{2}\right) \cdots K\left(x_{n-1}, x_{n}\right)$. In addition, let $\overleftarrow{x}=\left(x_{n}, x_{n-1}, \ldots, x_{0}\right)$. If (29) holds and $x_{0}=x_{n}$, then $\kappa(x)=\kappa(\overleftarrow{x})$.

Let $y$ be some other path ending at $y_{m}$. If (29) holds, $x_{0}=y_{0}, x_{n}=y_{m}$, and both paths have a positive probability of occurring, then

$$
\kappa(x) / \kappa(\overleftarrow{x})=\kappa(y) / \kappa(\overleftarrow{y})
$$

To see this, let $z$ be a path from $z_{0}=x_{n}=y_{m}$ and ending at $z_{\ell}=x_{0}=y_{0}$ with $\kappa(z)>0$. Then $\kappa(x z)=\kappa(x) \kappa(z)$ where $x z$ denotes the path that initially follows $x$ to $x_{n}$ and then follows $z$ back to $x_{0}$. Similarly, $\kappa(y z)=\kappa(y) \kappa(z)$. Under (29), $\kappa(x z)=\kappa(\overleftarrow{x z})=\kappa(\overleftarrow{z}) \kappa(\overleftarrow{x}) ;$ hence, $\kappa(x) / \kappa(\overleftarrow{x})=\kappa(\overleftarrow{z}) / \kappa(z)$. Similarly, $\kappa(y) / \kappa(\overleftarrow{y})=$ $\kappa(\overleftarrow{z}) / \kappa(z)$, which gives $(31)$.

Fix some state 0 and $\gamma(0)>0$. Let $x$ be a path from $x_{0}=0$ to some state $x_{n}$ with $\kappa(x)>0$. If (29) holds, then define $\gamma\left(x_{n}\right):=\gamma(0) \kappa(x) / \kappa(\overleftarrow{x})$. Under (29), it follows from (31) that the definition of $\gamma\left(x_{n}\right)$ makes sense since the r.h.s. is the same for all such paths. Consider a path that is one step longer: $x x_{n+1}$. For this path,

$$
\begin{aligned}
\gamma\left(x_{n+1}\right) & =\gamma(0) \frac{\kappa\left(x x_{n+1}\right)}{\kappa\left(\overleftarrow{\left.x x_{n+1}\right)}\right.} \\
& =\gamma(0) \frac{\kappa(x) K\left(x_{n}, x_{n+1}\right)}{K\left(x_{n+1}, x_{n}\right) \kappa(\overleftarrow{x})} \\
& =\gamma\left(x_{n}\right) \frac{K\left(x_{n}, x_{n+1}\right)}{K\left(x_{n+1}, x_{n}\right)},
\end{aligned}
$$

which means that (30) holds. Thus, (29) implies (30).

To see that (30) implies (29), suppose that $x$ is a path $x=\left(x_{0}, x_{1}, \ldots, x_{n}\right)$. Since $K\left(x_{j}, x_{j+1}\right)=\gamma\left(x_{j+1}\right) K\left(x_{j+1}, x_{j}\right) / \gamma\left(x_{j}\right)$, it follows that $\kappa(x)=\gamma\left(x_{n}\right) \kappa(\overleftarrow{x}) / \gamma\left(x_{0}\right)$. 
Letting $x_{0}=x_{n}$ shows that Kolmogorov's criterion holds.

To see that $\tilde{K}=\overleftarrow{K}$ implies reversibility, where both were computed using the same eigenvalue $t$,

$$
\begin{aligned}
\tilde{K}(x, y) & =\overleftarrow{K}(y, x) \\
\frac{K(x, y) h(y)}{t h(x)} & =\frac{\sigma(y) K(y, x)}{t \sigma(x)} \\
\frac{\sigma(x) K(x, y)}{h(x)} & =\frac{\sigma(y) K(y, x)}{h(y)}
\end{aligned}
$$

which means that (30) holds with $\gamma(x)=\sigma(x) / h(x)$.

The next claim that $\tilde{K}=\overleftarrow{K}$ under those conditions follows from a straightforward algebraic simplification.

If $K$ is reversible, then we know that (30) holds with $\gamma(x)$. Since $\sigma$ is $t$-invariant,

$$
\begin{aligned}
\overleftarrow{K}(x, y) & =\frac{\sigma(y) K(y, x)}{t \sigma(x)} \\
& =\frac{K(x, y) \sigma(y) / \gamma(y)}{t(\sigma(x) / \gamma(x))}
\end{aligned}
$$

Since the sum over the l.h.s. is $1, h=\sigma / \gamma$ is $t$-invariant, and $\tilde{K}=\overleftarrow{K}$.

If $K$ is reversible, then we know that (30) holds with $\gamma(x)$. Since $h$ is $t$-harmonic,

$$
\begin{aligned}
\tilde{K}(x, y) & =\frac{K(x, y) h(y)}{t h(x)} \\
& =\frac{\gamma(y) h(y) K(y, x)}{t \gamma(x) h(x)} .
\end{aligned}
$$

Since the sum over the l.h.s. is $1, \sigma=\gamma h$ is $t$-invariant, and $\tilde{K}=\overleftarrow{K}$

\section{One idea that yields a handful of identities}

We will exploit the following simple idea in computing various quantities of interest: frequently, it can be easier to analyze a well-chosen twist or time reversal rather than directly analyzing the process of interest. For example, suppose we are interested in computing the probability of ever hitting state $y$ starting from state $x$. If the wellchosen twist or time reversal eventually hits $y$ for certain when starting from $x$ - that is, if $\tilde{F}_{\xi^{*}}(x, y)=1$ or $\overleftarrow{F}_{\xi^{*}}(x, y)=1$ in (32) below-then we have the hitting probabilities for other twisted processes and time reversals. 
Initially, assume that the process of interest is a Markov chain starting in state $x$ that has transition matrix either $\overleftarrow{K}_{\xi}$ or $\tilde{K}_{\xi}$, where $\overleftarrow{K}_{\xi}$ would be the time reversal of $K$ with respect a $t$-invariant measure $\sigma_{\xi}$ and $\tilde{K}_{\xi}$ would be the time reversal with respect to $t$-harmonic function $h$. Assume that the quantities of interest are the probably of ever hitting $y$ starting from $x$, which will be denoted by $\overleftarrow{F}_{\xi}(x, y)$ and $\tilde{F}_{\xi}(x, y)$, respectively. Let either $\sigma_{\xi}^{*}$ be a (well-chosen) $t^{*}$-invariant measure, or let $h_{\xi}^{*}$ be a (well-chosen) $t^{*}$-harmonic function. For simplicity, assume $t^{*}=t$.

$$
\begin{aligned}
& \overleftarrow{K}_{\xi}(x, y)=\frac{\sigma_{\xi}(y)}{\sigma_{\xi^{*}}(y)} \frac{\sigma_{\xi^{*}(x)}}{\sigma_{\xi}(x)} \overleftarrow{K}_{\xi^{*}}(x, y) \text { and } \tilde{K}_{\xi}(x, y)=\tilde{K}_{\xi^{*}}(x, y) \frac{h_{\xi^{*}(x)}}{h_{\xi}(x)} \frac{h_{\xi}(y)}{h_{\xi^{*}}(y)} \\
& \overleftarrow{F}_{\xi}(x, y)=\frac{\sigma_{\xi}(y)}{\sigma_{\xi^{*}}(y)} \frac{\sigma_{\xi^{*}}(x)}{\sigma_{\xi}(x)} \overleftarrow{F}_{\xi^{*}}(x, y) \text { and } \tilde{F}_{\xi}(x, y)=\tilde{F}_{\xi^{*}}(x, y) \frac{h_{\xi^{*}(x)}}{h_{\xi}(x)} \frac{h_{\xi}(y)}{h_{\xi^{*}}(y)}
\end{aligned}
$$

The idea is simply to undo the twist or reverse and redo the twist or reverse with a better measure or harmonic function. In some contexts, it might be advantageous to mix the two: undoing the twist and then applying a time reversal or vice-versa.

The same idea can be exploited to obtain useful expressions for a different quantity of interest: the generating function $G_{(x, y)}(z)$ defined in (4). Suppose that $t=1 / z$ and that $\sigma_{\xi}^{*}$ is a (well-chosen) $t$-invariant measure or $h_{\xi}^{*}$ is a (well-chosen) $t$-harmonic function for $K$. Then

$$
\begin{aligned}
G_{(x, y)}(z) & =\frac{\sigma_{\xi^{*}}(y)}{\sigma_{\xi^{*}}(x)} \overleftarrow{G}_{\xi^{*}}(y, x) \\
& =\tilde{G}_{\xi^{*}}(x, y) \frac{h_{\xi^{*}}(y)}{h_{\xi^{*}}(x)}
\end{aligned}
$$

where $\overleftarrow{G}_{\xi^{*}}(y, x)$ is the expected number of visits to $x$ starting from $y$ for the reversed process with transition matrix $\overleftarrow{K}_{\xi^{*}}$ and $\tilde{G}_{\xi^{*}}(x, y)$ is the expected number of visits to $y$ starting from $x$ for the twisted process with transition matrix $\tilde{K}_{\xi^{*}}$. The proofs of the above equations are straightforward algebraic manipulations.

\section{Examples}

The examples are based on the Seneta and Vere-Jones' [27] semi-infinite random walk with absorption, which is the same as the gambler's ruin problem in the Introduction. Our primary example is a "hub-and-two-spoke example" that is depicted in Fig. 1. A hub-and-one-spoke model, shown in Fig. 2, functions as a notational bridge between 


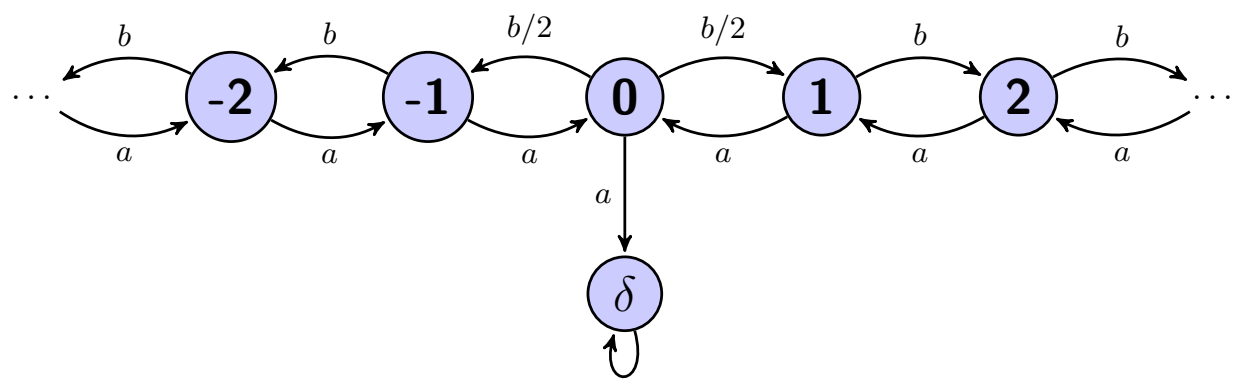

FiguRE 1: $K$ is restricted to $\mathbb{Z}$.

our primary example and the Seneta-Vere-Jones example. The hub-and-one-spoke example is simply a relabeled version of the Seneta-Vere-Jones example. The notation for these 3 examples will be the following:

Hub-and-two-spoke: Let $X=\left\{X_{0}, X_{1}, \ldots\right\}$ be a Markov chain with state space $\mathbb{Z}:=$ $\{\ldots,-1,0,1, \ldots\}$ augmented with an absorbing state $\delta$ and transition matrix $K$ between states in $\mathbb{Z}$ as shown in Figure 1.

Hub-and-one-spoke: Let $Y=\left\{Y_{0}, Y_{1}, \ldots\right\}$ denote the Markov chain with state space $\mathbb{N}_{0}$ augmented by an absorbing state $\delta$, and $Q$ will denote the transition matrix between states in $\mathbb{N}_{0}$ as shown in Figure 2.

Seneta-Vere-Jones: Let $Z=\left\{Z_{0}, Z_{1}, \ldots\right\}$ be the Markov chain with state space $\mathbb{N}$ augmented by an additional absorbing state 0 . The strictly substochastic matrix $P$ gives the transition probabilities between states in $\mathbb{N}$ where

$$
P=\left[\begin{array}{cccccc}
0 & b & 0 & 0 & 0 & \ldots \\
a & 0 & b & 0 & 0 & \ldots \\
0 & a & 0 & b & 0 & \ldots \\
\vdots & & & & &
\end{array}\right]
$$

Throughout, we assume that $0<b<1 / 2<a<1$ and $a+b=1$.

These three examples can be coupled in the following natural way. Given the huband-two-spoke model $X$, let $Y_{n}=\left|X_{n}\right|$ and $Z_{n}=\left(Y_{n}+1\right)$ for all $n$ prior to the (common) time of absorption $\zeta$. The coupling makes it easier to take advantage of results in Seneta-Vere-Jones [27]. For example, $K^{n}(0,0)=Q^{n}(0,0)=P^{n}(1,1)$ so 


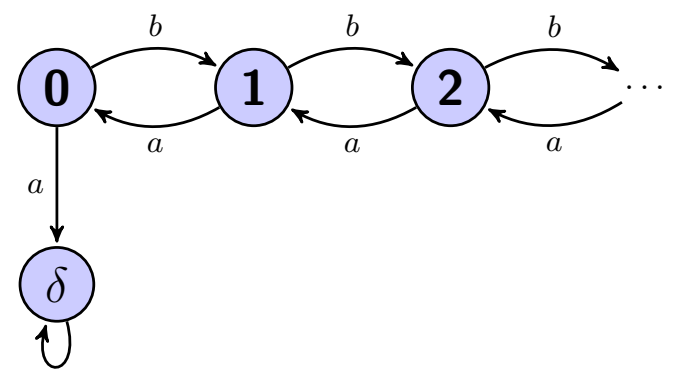

Figure 2: $Q$ is restricted to $\mathbb{N}_{0}$.

all three matrices have the same convergence parameter $R$, and from Seneta-VereJones [27] $R=1 / \rho$ where $\rho=2 \sqrt{a b}$. Next, we review a few results from Seneta-VereJones [27].

\subsection{Seneta-Vere-Jones semi-infinite random walk with absorption}

The matrix $P$ given in (35) for the Seneta-Vere-Jones example [27] is irreducible, strictly substochastic, and periodic with period 2 . Let $f_{n}$ be the probability that the first return to state 1 starting from 1 occurs at time $n$. Then the generating function $F(z)=\sum_{n \geq 0} f_{n} z^{n}=\left(1-\sqrt{1-4 a b z^{2}}\right) / 2$. Hence, the convergence parameter of $P$ is $R=1 / \rho$ where $\rho=2 \sqrt{a b}$. Since $F(R)=1 / 2, G_{1,1}(R)=1 /(1-F(R))=2<\infty, P$ must be $R$-transient.

Seneta and Vere-Jones [27] prove a periodic Yaglom limit where the $\rho$-invariant QSD on the r.h.s. is $\pi^{*}$ given in (1), which does not depend on the starting state $x$. From (35) in [27] and from p. 430 of [27], we have the following asymptotic expressions as $n \rightarrow \infty$

$$
\begin{aligned}
P^{2 n}(x, y) & \sim x\left(\sqrt{\frac{a}{b}}\right)^{x-1} y\left(\sqrt{\frac{b}{a}}\right)^{y-1} \sqrt{\frac{1}{\pi}} \frac{(4 a b)^{n}}{n^{3 / 2}} & & \text { for } y-x \text { even } \\
\mathbb{P}\left\{\zeta=n \mid Z_{0}=x\right\} & =\frac{x}{n}\left(\begin{array}{c}
n \\
(n-x) / 2
\end{array}\right) b^{(n-x) / 2} a^{(n+x) / 2} & & \text { for } n-x \text { even } \\
& \sim \frac{x \cdot 2^{n+1}}{(2 \pi)^{1 / 2}(n)^{3 / 2}} b^{\frac{1}{2}(n-x)} a^{\frac{1}{2}(n+x)} & & \text { for } n-x \text { even }
\end{aligned}
$$

where $\zeta$ is the time of absorption. 


\subsection{Hub-and-two-spoke model: our primary example}

At first, this two-spoke variation may seem pointless, but the point is to construct a tractable model that has more than one way to escape from 0 . Each spoke provides a different escape route. We will show that the periodic Yaglom limit starting from state $x$ in the hub-and-two-spoke example is

$$
\pi_{x}(y)= \begin{cases}\frac{1-\rho}{2 a}\left(1+|y|+\frac{x}{1+|x|} y\right)\left(\sqrt{\frac{b}{a}}\right)^{|y|} & \text { for } y \in \mathbb{Z} \backslash\{0\} \\ \frac{1-\rho}{a} & \text { for } y=0 .\end{cases}
$$

For $y>0, \pi_{x}(y)$ is strictly increasing in $x$ - each starting state $x$ has a different Yaglom limit. For aperiodic examples, it suffices to look at either the even states or the odd states and use the two-step transition matrix $K^{2}$. For $K^{2}$, the limiting conditional distribution of being in state $2 y$ for $y>0$ is strictly increasing in the starting state $2 x$.

Theorem 1. The hub-and-two-spoke model with $0<b<1 / 2<a<1$ and $a+b=$ 1 is periodic with period $d=2$ and has a periodic Yaglom limit $\pi_{x}$ given in (38). Equivalently,

$$
\begin{aligned}
\frac{K^{2 n}(x, y)}{K^{2 n}(x, S)} & \rightarrow \frac{\pi_{x}(y)}{\pi_{x}(2 \mathbb{Z})} \quad \text { for } x \text { even and } y \text { even }, \\
\frac{K^{2 n+1}(x, y)}{K^{2 n+1}(x, S)} & \rightarrow \frac{\pi_{x}(y)}{\pi_{x}(2 \mathbb{Z}+1)} \quad \text { for } x \text { even and } y \text { odd }, \\
\frac{K^{2 n}(x, y)}{K^{2 n}(x, S)} & \rightarrow \frac{\pi_{x}(y)}{\pi_{x}(2 \mathbb{Z}+1)} \quad \text { for } x \text { odd and } y \text { odd }, \\
\frac{K^{2 n+1}(x, y)}{K^{2 n+1}(x, S)} & \rightarrow \frac{\pi_{x}(y)}{\pi_{x}(2 \mathbb{Z})} \quad \text { for } x \text { odd and } y \text { even }
\end{aligned}
$$

where

$$
\begin{aligned}
\pi_{x}(2 \mathbb{Z}) & =\frac{1}{1+\rho} \\
\pi_{x}(2 \mathbb{Z}+1) & =\frac{\rho}{1+\rho} .
\end{aligned}
$$

In the next two sections, we prove Theorem 1. To show that (39)-(40) hold, we first look at the asymptotics of their denominators, and then the asymptotics for their numerators. In the remainder of this section, we describe the $\rho$-invariant measures and harmonic functions for the hub-and-two-spoke model. 
We leave it to the reader to show that the matrix $K$ possesses a family of $\rho$-invariant QSDs $\sigma_{\xi}$ indexed by $\xi \in[-1,1]$ and given by

$$
\sigma_{\xi}(y)= \begin{cases}\frac{1-\rho}{2 a}(1+|y|+\xi y)\left(\sqrt{\frac{b}{a}}\right)^{|y|} & \text { for } y \in \mathbb{Z} \backslash\{0\} \\ \frac{1-\rho}{a} & \text { for } y=0 .\end{cases}
$$

Thus, if the chain starts from state $x, \pi_{x}=\sigma_{\xi}$ with $\xi=x /(1+|x|)$ is the periodic Yaglom limit. The derivation of the asymptotics of $K$ is given in the next sections.

By looking at $\sigma_{\xi}(1)$, it is clear that every member of the family is a different distribution. For each distribution, the correct amount of mass is lost to absorption: $a \sigma_{\xi}(0)=$ $1-\rho$. As $\xi$ increases from -1 to $1, \sigma_{\xi}(|y|)$ increases from $((1-\rho) /(2 a))(\sqrt{b / a})^{|y|}$ to $((1-\rho) /(2 a))(1+2|y|)(\sqrt{b / a})^{|y|}$. When $\xi=0$, the distribution is symmetric with $\sigma_{0}(y)=\sigma_{0}(-y)$. The mass $\sigma_{\xi}(y)+\sigma_{\xi}(-y)=\pi^{*}(|y|+1)$ does not depend on $\xi ; \pi^{*}$ was defined in (1). Consequently, the mass on the even integers $2 \mathbb{Z}$ and the odd integers $2 \mathbb{Z}+1$ does not depend on $\xi$. Since

$$
\begin{array}{ll}
\sigma_{\xi} K(2 \mathbb{Z})=\rho \sigma_{\xi}(2 \mathbb{Z}) & \text { by } \rho \text {-invariance, and } \\
\sigma_{\xi} K(2 \mathbb{Z})=\sigma_{\xi}(2 \mathbb{Z}+1) & \text { by periodicity and nonabsorption, }
\end{array}
$$

we have

$$
\sigma_{\xi}(2 \mathbb{Z})=\frac{1}{1+\rho}, \sigma_{\xi}(2 \mathbb{Z}+1)=\frac{\rho}{1+\rho}
$$

In addition to the $\rho$-invariant measures $\sigma_{\xi}, K$ also has $\rho$-harmonic functions $h_{\xi}$. A function $h \geq 0$, which we think of as a column vector with elements $h(y)$ for $y \in S$, is $\rho$-harmonic if $K h=\rho h$.

Equivalently, $h$ is a nonnegative right eigenvector for the eigenvalue $\rho=1 / R$. In this example, $K$ has a family of nonnegative $\rho$-harmonic functions $h_{\xi}$ indexed by $\xi \in[-1,1]$ :

$$
h_{\xi}(y):=[1+|y|+\xi y]\left(\sqrt{\frac{a}{b}}\right)^{|y|} \text { for } y \in \mathbb{Z} .
$$

Proposition 8. All $\rho$-invariant probability measures for the hub-and-two-spokes example are in the family (41) and all positive $\rho$-harmonic functions are in the family (42). 
Proof. To see that there are no other positive $\rho$-harmonic functions $h$ with $h(0)=1$, notice that $h(y)$ satisfies the difference equation $b h(y+1)-\rho h(y)+a h(y-1)=0$ for $y \in \mathbb{N}$. Since this difference equation is a linear, homogeneous, second order difference equation with constant coefficients, we look at the roots of the characteristic equation $b r^{2}-\rho r+a$ to determine the general solution. Both roots are $r=\sqrt{a / b}$, so $h(y)=$ $\left(c_{1}+c_{2} y\right) r^{y}$ spans the space of all solutions. Since $h(0)=1$, we must have $c_{1}=1$, which we do in (42). For $h(y)$ to be nonnegative, we need $c_{2} \geq 0$. For $y \geq 0$, that means that $(1+\xi)=c_{2} \geq 0$ implying $\xi \geq-1$. By symmetry, we need $\xi \leq 1$ so that $h(y)$ is nonnegative for $y<0$. Thus, (42) is the set of all positive harmonic functions normalized to have $h(0)=1$. A similar difference equation argument shows that $\sigma_{\xi}$ for $\xi \in[-1,1]$ describes all $\rho$-invariant measures up to scalar multiples.

In section 3 we described a duality that sometimes exists between $\rho$-invariant measures and $\rho$-harmonic functions. We now show that for each value of $\xi \in[-1,1]$ the $\rho$-harmonic function $h_{\xi}$ and the $\rho$-invariant measure $\sigma_{\xi}$ are linked. For the hub-andtwo-spokes example, the measure

$$
\gamma(x)= \begin{cases}\frac{1-\rho}{2 a}\left(\frac{b}{a}\right)^{|x|} & \text { for } x \neq 0 \\ \frac{1-\rho}{a} & \text { for } x=0\end{cases}
$$

satisfies $\gamma(x) K(x, y)=\gamma(y) K(y, x)$. From Prop. 7, the $\rho$-invariant measure linked with the $\rho$-harmonic function $h_{\xi}(x)$ would be $h_{\xi}(x) \gamma(x)$, which simplifies to $\sigma_{\xi}(x)$.

5.2.1. Asymptotic behavior of the survival probability. For $x$ even, $K^{2 n}(x, S)=K^{2 n-1}(x, S)$.

Since $K^{n}(-x, S)=K^{n}(x, S)$, also assume that $x$ is nonnegative. Now,

$$
\begin{aligned}
K^{2 n}(x, S) & =\mathbb{P}\left\{\zeta>2 n \mid X_{0}=x\right\} \\
& =\sum_{k=1}^{\infty} \mathbb{P}\left\{\zeta=2 n+2 k-1 \mid X_{0}=x\right\} \\
& =\sum_{k=1}^{\infty} \mathbb{P}\left\{\zeta=2 n+2 k-1 \mid Z_{0}=x+1\right\}
\end{aligned}
$$


Since $\mathbb{P}\left\{\zeta=2 n+2 k-1 \mid Z_{0}=x+1\right\}$ is asymptotically equivalent to the r.h.s. of (37) after replacing $n$ by $2 n+2 k-1$ and $x$ by $x+1$ and since the equivalence is uniform over $k \in \mathbb{N}$ (for a discussion of this condition, see (5.27) of [22]),

$$
\begin{aligned}
K^{2 n}(x, S) & \sim \sum_{k=1}^{\infty} \frac{(x+1) 2^{2 n+2 k}}{(2 \pi)^{1 / 2}(2 n+2 k-1)^{3 / 2}} b^{(2 n+2 k-x-2) / 2} a^{(2 n+2 k+x) / 2} \\
& =\frac{(x+1)}{(2 \pi)^{1 / 2}}\left(\sqrt{\frac{a}{b}}\right)^{x} \frac{(4 a b)^{n}}{(2 n)^{3 / 2}} \frac{1}{b} \sum_{k=1}^{\infty} \frac{(4 a b)^{k}}{(1+(2 k-1) /(2 n))^{3 / 2}} \\
& \sim \frac{(x+1)}{(2 \pi)^{1 / 2}}\left(\sqrt{\frac{a}{b}}\right)^{x} \frac{(4 a b)^{n}}{(2 n)^{3 / 2}} \frac{4 a}{1-4 a b} \quad \text { for even } x \geq 0 \\
& =(x+1)\left(\sqrt{\frac{a}{b}}\right)^{x} \frac{a}{1-4 a b} \frac{1}{\sqrt{\pi}} \frac{(4 a b)^{n}}{n^{3 / 2}} \quad \text {. }
\end{aligned}
$$

where the second from last step follows from using dominated convergence to show that

$$
\lim _{n \rightarrow \infty} \sum_{k=1}^{\infty} \frac{(4 a b)^{k}}{(1+(2 k-1) /(2 n))^{3 / 2}}=\sum_{k=1}^{\infty}(4 a b)^{k} .
$$

5.2.2. The periodic Yaglom limit of the hub-and-two-spoke model. In this section, we show $K$ corresponding to Figure 1 has a periodic Yaglom limit; that is, we establish (38). Let $x, y \geq 0$. From the coupling, we have

$$
Q^{n}(x, y)=K^{n}(x,-y)+K^{n}(x, y)
$$

and we can determine the asymptotics of $Q$ from the asymptotics of $P$ given in (36) that were derived in Seneta and Vere-Jones [27].

Similar to the classical ballot problem, there are two types of paths from $x$ to $y$ : those that visit 0 and those that do not. From the reflection principle, any path from $x$ to $y$ that visits 0 has a corresponding path from $-x$ to $y$ with the same probability of occurring. Thus, if ${ }_{\{0\}} K^{n}(x, y)$ denotes the probability of going from $x$ to $y$ in $n$ steps without visiting zero in between, we have

$$
K^{n}(x, y)={ }_{\{0\}} K^{n}(x, y)+K^{n}(-x, y)={ }_{\{0\}} K^{n}(x, y)+K^{n}(x,-y) .
$$

From the coupling, ${ }_{\{0\}} K^{n}(x, y)=P^{n}(x, y)$.

For $x \geq 0$ and $y \geq 1$,

$$
Q^{n}(x, y)=K^{n}(x, y)+K^{n}(x,-y)
$$


Hence

$$
\begin{aligned}
K^{n}(x, y) & =Q^{n}(x, y)-K^{n}(x,-y) \\
& =Q^{n}(x, y)-\left[K^{n}(x, y)-{ }_{\{0\}} K^{n}(x, y)\right] \\
& =\frac{Q^{n}(x, y)+{ }_{\{0\}} K^{n}(x, y)}{2} .
\end{aligned}
$$

Similarly,

$$
K^{n}(x,-y)=\frac{Q^{n}(x, y)-{ }_{\{0\}} K^{n}(x, y)}{2} .
$$

5.2.3. Yaglom limits from even states to even. If either $x$ is 0 or $y$ is 0 , then the asymptotics of $K^{n}(x, y) / K^{n}(x, S)$ can be obtained directly from the results in Seneta and Vere-Jones [27] through the coupling Thus, (13) holds for $k=0$.

Now, let $x, y \geq 1$ and even. From the couplings,

$$
Q^{2 n}(x, y)=P^{2 n}(x+1, y+1),{ }_{\{0\}} K^{2 n}(x, y)=P^{2 n}(x, y)
$$

Since $x-y$ is also even, (36) gives

$$
\begin{aligned}
Q^{2 n}(x, y) & \sim(x+1)\left(\sqrt{\frac{a}{b}}\right)^{x}(y+1)\left(\sqrt{\frac{b}{a}}\right)^{y} \frac{1}{\sqrt{\pi}} \frac{(4 a b)^{n}}{n^{3 / 2}} \\
{ }_{\{0\}} K^{2 n}(x, y) & \sim x\left(\sqrt{\frac{a}{b}}\right)^{x-1} y\left(\sqrt{\frac{b}{a}}\right)^{y-1} \frac{1}{\sqrt{\pi}} \frac{(4 a b)^{n}}{n^{3 / 2}}
\end{aligned}
$$

Hence, using (45) and (44),

$$
\begin{aligned}
\frac{K^{2 n}(x, y)}{K^{2 n}(x, S)} \sim & \frac{(x+1)\left(\sqrt{\frac{a}{b}}\right)^{x}(y+1)\left(\sqrt{\frac{b}{a}}\right)^{y} \frac{1}{\sqrt{\pi}} \frac{(4 a b)^{n}}{n^{3 / 2}}}{2(x+1)\left(\sqrt{\frac{a}{b}}\right)^{x} \frac{a}{1-4 a b} \frac{1}{\sqrt{\pi}} \frac{(4 a b)^{n}}{n^{3 / 2}}} \\
& +\frac{x\left(\sqrt{\frac{a}{b}}\right)^{x-1} y\left(\sqrt{\frac{b}{a}}\right)^{y-1} \frac{1}{\sqrt{\pi}} \frac{(4 a b)^{n}}{n^{3 / 2}}}{2(x+1)\left(\sqrt{\frac{a}{b}}\right)^{x} \frac{a}{1-4 a b} \frac{1}{\sqrt{\pi}} \frac{(4 a b)^{n}}{n^{3 / 2}}} \\
& =\frac{1-4 a b}{2 a}\left[(y+1)+\frac{x y}{x+1}\right]\left(\sqrt{\frac{b}{a}}\right)^{y} \\
= & \frac{1-\rho^{2}}{2 a}\left(1+y+\frac{x y}{x+1}\right)\left(\sqrt{\frac{b}{a}}\right)^{y} .
\end{aligned}
$$


We now argue that (13) holds for $d=2, k=0$ and $S_{0}=2 \mathbb{Z}$ with

$$
\pi_{x}^{0}(y)= \begin{cases}\frac{1-\rho^{2}}{2 a}\left(1+|y|+\frac{x y}{|x|+1}\right)\left(\sqrt{\frac{b}{a}}\right)^{|y|} & \text { for } y \neq 0 \text { and even } x, y \\ \frac{1-\rho^{2}}{a} & \text { for } y=0 \text { and even } x\end{cases}
$$

We are still assuming even $x, y \geq 1$. Since $\pi_{x}^{0}(y)$ is the same as (48), we know that (49) holds for even $x, y \geq 1$. The asymptotics for $-x$ to $-y$ are the same as from $x$ to $y$, and $\pi_{-x}^{0}(-y)=\pi_{x}^{0}(y)$ so (49) also holds in this case.

To handle from $x$ to $-y$, we use (46) instead of (45), which causes a single sign change, and the final result agrees with (49) in this case. The asymptotics from $-x$ to $y$ are the same as from $x$ to $-y$, and (49) gives the same result in both cases.

\subsubsection{Yaglom limits from even states to odd. Instead of using a similar argument for} the asymptotics from even to odd, we use Proposition 3. Since (13) holds for $k=0$, Proposition 3 gives (13) for $k=1$. Proposition 3 also gives $\pi_{x}^{1}$, which is a probability measure on the odd states $S_{1}=2 \mathbb{Z}-1$ giving the asymptotics from even to odd. Next, Proposition 3 gives us the $\rho$-invariant QSD $\pi_{x}$ for every even $x$. We leave it to the reader to show that $\pi_{x}$ is given by (38). Hence, we have the asymptotics going to any state as long as the starting state is even.

5.2.5. Yaglom limits starting from odd states. To finish determining the periodic Yaglom limit, we obtain the asymptotics starting from an odd state. Instead of direct calculations like those that led to (47), We use Prop. 4. To do so, we need the function $\hat{h}_{0}$ defined in (21). The class $S_{0}$ is the even states. Choose $x_{0}=0$ to be the reference state. From (44) and symmetry, it follows that $\hat{h}_{0}(x)=(|x|+1)(\sqrt{a / b})^{|x|}$. Since the assumptions of Prop. 6 hold, there exists a $\rho$-harmonic function $\hat{h}$ that agrees with $\hat{h}_{0}$ on $S_{0}$ such that (28) holds. However, (42) describes all $\rho$-harmonic functions for $K$. The only $\rho$-harmonic function that could agree with $\hat{h}$ on $S_{0}$ is $h_{0}$, that is, $h_{\xi}$ with $\xi=0$ in (42). Thus, $\hat{h}=h_{0}$. Furthermore, since the assumptions of Prop. 6 hold, the denominator of (24) simplifies to $\rho^{d-j} \hat{h}(u)$.

Let $u \in S_{1}$, that is, an odd state, and $y \in S_{0}$. To make things easier, temporarily 
assume $u \geq 1$ and $y \neq 0$. From (23),

$$
\begin{aligned}
\pi_{u}^{-1}(y) & =\sum_{x \in S_{0}} w_{u, x} \pi_{x}^{0}(y) \\
& =\frac{a \hat{h}(u-1)}{\rho \hat{h}(u)} \pi_{u-1}^{0}(y)+\frac{b \hat{h}(u+1)}{\rho \hat{h}(u)} \pi_{u+1}^{0}(y) \\
& =\frac{a u}{2 \sqrt{a b}(u+1)} \sqrt{\frac{b}{a}} \pi_{u-1}^{0}(y)+\frac{b(u+2)}{2 \sqrt{a b}(u+1)} \sqrt{\frac{a}{b}} \pi_{u+1}^{0}(y) \\
& =\frac{u}{2(u+1)} \pi_{u-1}^{0}(y)+\frac{u+2}{2(u+1)} \pi_{u+1}^{0}(y) \\
& =\frac{1-\rho^{2}}{2 a}\left(1+|y|+\left[\frac{u}{2(u+1)} \frac{u-1}{u}+\frac{u+2}{2(u+1)} \frac{u+1}{u+2}\right] y\right)\left(\sqrt{\frac{b}{a}}\right)^{|y|} \\
& =\pi_{u}^{0}(y)
\end{aligned}
$$

where we used (49) several times. The case with $y=0$ is much simpler and also simplifies to $\pi_{u}^{0}(0)$. We leave the cases with $u \leq 1$ to the reader. Thus, we have the asymptotics starting from an odd state and going to an even state. Again, Prop. 3 allows us to extend the result to going to any state. Hence, we have the asymptotics starting from any state and going to any state. Combining all of the results, we have that (11) holds where $\pi_{u}(y)$ is given in (38) (though we would have to interchange labels on $S_{0}$ and $S_{1}$ in (11) if the initial state were odd).

5.2.6. Rates of convergence and the starting state's influence. One fear with Yaglom limits is that by the time the transient conditional distribution becomes close to the limiting conditional distribution, the probability of non-absorption will be so small that the limit will be of little practical importance. To briefly address this fear, we describe some empirical results where the dependence of the limiting distribution on the initial state is apparent after a small number of steps (50 steps in Table 1) and the non-absorption probability is not ridiculously small (0.00047 in Table 1).

Suppose that $b=1 / 5$, so $a=4 / 5$ and $\rho=4 / 5$. Also assume that the initial state is $X_{0}=10$. From (49), $\mathbb{P}_{10}\left\{X_{2 n}=0 \mid \zeta>2 n\right\} \rightarrow \pi_{10}^{0}(0)=0.45$ and $\mathbb{P}_{10}\left\{X_{2 n} \in 2 \mathbb{N} \mid \zeta>\right.$ $2 n\} \rightarrow 201 / 440 \approx 0.46$. The latter limit is the limiting conditional probability starting from 10 of being a strictly positive, even integer after an even number of steps. Starting from state $10, \xi=10 / 11$; asymptotically after a large, even number of steps, over $90 \%$ of the probability mass is on the nonnegative, even integers. The remaining probability 


\begin{tabular}{rcccc}
\hline$n$ & $\frac{K^{n}(10,0)}{K^{n}(10, S)}$ & $\frac{K^{n}(10,2 \mathbb{N})}{K^{n}(10, S)}$ & $\frac{K^{n}(10,-2 \mathbb{N})}{K^{n}(10, S)}$ & $K^{n}(10, S)$ \\
\hline 0 & 0.00 & 1.00 & 0.00 & 1.00 \\
10 & 0.11 & 0.89 & 0.00 & 1.00 \\
20 & 0.38 & 0.60 & 0.02 & 0.31 \\
30 & 0.44 & 0.53 & 0.03 & 0.042 \\
40 & 0.46 & 0.50 & 0.04 & 0.0050 \\
50 & 0.46 & 0.49 & 0.05 & 0.00047 \\
$\infty$ & 0.45 & 0.46 & 0.09 & 0.0 \\
\hline
\end{tabular}

TABLE 1: Rates of convergence starting from state 10 with $b=1 / 5$ and $2 \mathbb{N}=\{2,4,6, \ldots\}$.

mass, approximately 0.09 , is on the strictly negative, even integers. Table 1 suggests that at least in some cases the limiting conditional distribution might be giving some information before the probability of non-absorption $K^{n}(10, S)$ becomes ridiculously smal1.

Table 1 also illustrates the long range influence of the starting state. The limiting conditional probability of being in a strictly positive state is roughly 5 times larger than the limiting conditional probability of being in a strictly negative state. If the initial state had been zero, then the two limiting conditional probabilities would have been equal. If the process had started in state -10 , the third and fourth columns would swap.

5.2.7. Domain of attraction paradox. The domain of attraction problem is to determine which initial distributions lead to a particular QSD describing the limiting conditional behavior. To make things concrete, consider our hub-and-two-spoke example in Fig. 1. Suppose $b=1 / 5$ and $X_{0}=6$, which means that $\pi_{6}$ describes the limiting conditional distribution. Then, $X_{2}$ has distribution $K^{2}(6, \cdot)$, which is 8 with probability $b^{2}, 6$ with probability $2 a b$, and 4 with probability $a^{2}$. It might seem obvious that $K^{2}(6, \cdot)$ must be in the domain of attraction of $\pi_{6}$, but it is not even true. The limiting conditional behavior is quite different when these two distributions are used as initial distributions even though there is no possibility of absorption in 2 steps when starting from state 6 .

We will show below that an initial distribution with support on $\{4,6,8\}$ that is in the 
domain of attraction of $\pi_{6}$ is 8 with probability $b^{2}(225 / 28)=9 / 28,6$ with probability $2 a b(25 / 16)=1 / 2$, and 4 with probability $a^{2}(125 / 448)=5 / 28$. This distribution is obtained by letting the mass at $y$ be

$$
\frac{K^{n d}(6, y) \hat{h}(y)}{\sum_{z} K^{n d}(6, z) \hat{h}(z)},
$$

where $n d=2$. Intuition for this choice is given in Remark 6 .

For our hub-and-two-spoke example, we can do much more than locate a few distribution within the domain of attraction of $\pi_{x}$; we can give a fairly complete solution to the domain of attraction problem when the initial distribution has a finite support. Initially, assume that the support of $X_{0}$ is either on the odd or even integers. If the support of $X_{0}$ is finite and concentrated on either the evens or odds, then the limiting conditional behavior of the hub-and-two-spoke model is described by $\sigma_{\xi}$ where $\xi=\mathbb{E}\left[X_{0} /\left(\left|X_{0}\right|+1\right)\right]$. By this we mean

$$
\lim _{n \rightarrow \infty} \mathbb{P}\left\{X_{n d+k}=y \mid X_{n d+k} \in S\right\}=\frac{\sigma_{\xi}(y)}{\sigma_{\xi}\left(S_{k}\right)} .
$$

To see that (50) holds, Let $\phi(x)=\mathbb{P}\left\{X_{0}=x\right\}$, and let $S_{0}$ denote the class that includes the support of $\phi$. Notice that $\pi_{x}\left(S_{k}\right)$ is a constant for all $x \in S_{0}$ so that $\sigma_{\xi}\left(S_{k}\right)=\pi_{x}\left(S_{k}\right)$. Since we have a periodic Yaglom limit from each state $x$, we know that (11) holds. Hence,

$$
\begin{aligned}
\lim _{n \rightarrow \infty} \sum_{x} \phi(x) \mathbb{P}_{x}\left\{X_{n d+k}=y \mid X_{n d+k} \in S\right\} & =\sum_{x} \phi(x) \frac{\pi_{x}(y)}{\pi_{x}\left(S_{k}\right)} \\
& =\frac{1}{\pi_{x}\left(S_{k}\right)} \sum_{x} \phi(x) \sigma_{x /(|x|+1)}(y) \\
& =\frac{\sigma_{\xi}(y)}{\sigma_{\xi}\left(S_{k}\right)}
\end{aligned}
$$

where $\xi=\mathbb{E}\left[X_{0} /\left(\left|X_{0}\right|+1\right)\right]$, and we used the finite support to justify interchanging the limit and sum.

If the initial distribution were $K^{2}(6, \cdot)$ with $b=1 / 5$, then the limiting conditional behavior would be described by $\sigma_{\xi}$ where $\xi=6472 / 7875$. However, if the initial distribution is on 4,6 , and 8 with probabilities $5 / 28,1 / 2$, and $9 / 28$, resp., then $\mathbb{E}\left[X_{0} /\left(\left|X_{0}\right|+1\right)\right]=6 / 7$, and $\sigma_{6 / 7}=\pi_{6}$.

Thus, we know that all distributions with a finite support concentrated on either the odd or the even integers and having $\xi=\mathbb{E}\left[X_{0} /\left(\left|X_{0}\right|+1\right)\right]$ are in the domain of 
attraction of $\sigma_{\xi}$. The case where the initial distribution has a finite support that includes both even and odd integers is now a fairly straightforward mixture of what we have just done. We leave the details, starting with the appropriate expression for the r.h.s. of (50), to the reader.

5.2.8. Harmonic functions arising from ratio limits. Suppose $x, y$ are both even integers. From the asymptotic expression for $K^{2 n}$, it follows that

$$
\frac{K^{2 n}(x, y)}{K^{2 n}(0, y)} \rightarrow \frac{h_{\xi}(x)}{h_{\xi}(0)}=h_{\xi}(x)
$$

where $h_{\xi}$ is the $\rho$-harmonic function given in (42) with $\xi=y /(|y|+1)$. Similarly, for $x$ odd and $y$ even integers

$$
\frac{K^{2 n+1}(x, y)}{K^{2 n}(0, y)} \rightarrow \rho \frac{h_{\xi}(x)}{h_{\xi}(0)} .
$$

We leave the other cases to the reader.

The above results are not surprising given the existence of the measure $\gamma$ described in Prop. 7. For example if $x, y$ are both even integers then

$$
\begin{aligned}
\frac{K^{2 n}(x, y)}{K^{2 n}(0, y)} & =\frac{\gamma(0)}{\gamma(x)} \frac{K^{2 n}(y, x)}{K^{2 n}(y, 0)} \\
& \rightarrow \frac{\gamma(0)}{\gamma(x)} \frac{\pi_{y}^{0}(x)}{\pi_{y}^{0}(0)} \\
& =\frac{\gamma(0)}{\pi_{y}(0)} \frac{\pi_{y}(x)}{\gamma(x)} \\
& =\frac{h_{\xi}(x)}{h_{\xi}(0)} .
\end{aligned}
$$

5.2.9. Time reversals and $h$-transforms. In addition to the $\rho$-invariant measures for the hub-and-two-spoke example, we have determined all nonnegative $\rho$-harmonic functions for $K$. Since we have a multitude of $\rho$-invariant measures $\sigma_{\xi}$ and $\rho$-harmonic functions $h_{\xi}$, we can define a multitude of time reversals

$$
\overleftarrow{K}_{\xi}(x, y)=\frac{R \sigma_{\xi}(y) K(y, x)}{\sigma_{\xi}(x)}
$$

and a multitude of twisted processes (tilted processes, Doob's $h$-transform, DermanVere-Jones transform, change-of-measure)

$$
\tilde{K}_{\xi}(x, y)=\frac{R K(x, y) h_{\xi}(y)}{h_{\xi}(x)}
$$


Both $\overleftarrow{K}_{\xi}$ and $\tilde{K}_{\xi}$ are stochastic matrices on $S$ for every $\xi \in[-1,1]$. However, depending on the choice of $\xi$, the time reversal's behavior can vary considerably, and similarly for the twisted process. In the hub-and-two-spoke example, we have

$$
\mathbb{P}_{0}\left\{X_{1}=1 \mid X_{1} \in S\right\}=\frac{K(0,1)}{K(0, S)}=\frac{1}{2}
$$

but, depending on the choice of $\xi, \tilde{K}_{\xi}(0,1)$ and $\overleftarrow{K}_{\xi}(0,1)$ can take any value in the interval $[1 / 4,3 / 4]$. Even if the time until absorption is $\zeta>n$ and $n$ is tending to $\infty$, the initial state $x$ still influences the state prior to absorption $X_{\zeta-1}$, and two steps prior to absorption, $X_{\zeta-2}, \ldots$

5.2.10. Escape probabilities for time reversals and $h$-transforms. Let $\overleftarrow{X}^{\xi}=\left(\overleftarrow{X}_{0}^{\xi}, \overleftarrow{X}_{1}^{\xi}, \ldots\right)$ denote a Markov chain with transition matrix $\overleftarrow{K}_{\xi}$ as given in (51). The transition matrix $\overleftarrow{K}_{\xi}$ is a birth-death chain on the integers that is stochastic and transient, so $\overleftarrow{X}^{\xi}$ must escape to either plus infinity or negative infinity. We now compute the probability of escaping to plus infinity starting from $\mathrm{x}$; that is, we compute $\overleftarrow{h}_{\xi}(x):=\mathbb{P}\left\{\overleftarrow{X}^{\xi}\right.$ escapes to $\left.+\infty \mid \overleftarrow{X}_{0}^{\xi}=x\right\}$

Computing $\overleftarrow{h}_{\xi}(x)$ is easiest in the two extreme cases. When $\xi=1, \overleftarrow{K}_{1}$ on the negative integers is a symmetric, simple random walk: for $x<0$

$$
\begin{aligned}
\overleftarrow{K}_{1}(x, x+1) & =\frac{\sigma_{1}(x+1) K(x+1, x)}{\rho \sigma_{1}(x)} \\
& =\frac{((1-\rho) / 2 a)(\sqrt{b / a})^{|x+1|} b}{\rho((1-\rho) / 2 a)(\sqrt{b / a})^{|x|}} \\
& =\frac{b}{2 \sqrt{a b} \sqrt{b / a}} \\
& =1 / 2
\end{aligned}
$$

which means that the process cannot escape to negative infinity. Hence, $\overleftarrow{h}_{1}(x)=1$ Similarly, $\overleftarrow{h}_{-1}(x)=0$

Now, we can handle the more interesting cases with $-1<\xi<1$. From the first equation in (32) with $\xi^{*}=1$,

$$
\overleftarrow{F}_{\xi}(x, \ell)=\frac{\sigma_{\xi}(\ell)}{\sigma_{1}(\ell)} \frac{\sigma_{1}(x)}{\sigma_{\xi}(x)} \overleftarrow{F}_{1}(x, \ell)
$$


Since $\overleftarrow{F}_{1}(x, \ell)=1$ whenever $\ell>x$ and since the walk is nearest neighbor and transient letting $\ell \rightarrow \infty$ gives the desired escape probability

$$
\overleftarrow{h}_{\xi}(x)=\frac{1+\xi}{2} \frac{\sigma_{1}(x)}{\sigma_{\xi}(x)}
$$

which can be rewritten as

$$
\sigma_{\xi}(x) \overleftarrow{h}_{\xi}(x)=\frac{1+\xi}{2} \sigma_{1}(x)
$$

By considering the other extreme case with $\xi^{*}=-1$ and letting $\ell \rightarrow-\infty$, we obtain

$$
\sigma_{\xi}(x)\left(1-\overleftarrow{h}_{\xi}(x)\right)=\frac{1-\xi}{2} \sigma_{-1}(x)
$$

Adding (53) and (54) yields the representation

$$
\sigma_{\xi}(y)=\frac{1+\xi}{2} \sigma_{1}(y)+\frac{1-\xi}{2} \sigma_{-1}(y) \quad \text { for } \xi \in[0,1] .
$$

Now, we turn our attention to escape probabilities for the twisted processes. Let $\tilde{X}^{\xi}=\left(\tilde{X}_{0}^{\xi}, \tilde{X}_{1}^{\xi}, \ldots\right)$ denote a Markov chain with transition matrix $\tilde{K}_{\xi}$ as given in (52). The transition matrix $\tilde{K}_{\xi}$ is a birth-death chain on the integers that is stochastic and transient, so $\tilde{X}^{\xi}$ must escape to either plus infinity or negative infinity. We now compute the probability of escaping to plus infinity starting from $\mathrm{x}$; that is, we compute $\tilde{h}_{\xi}(x):=\mathbb{P}\left\{\tilde{X}^{\xi}\right.$ escapes to $\left.+\infty \mid \tilde{X}_{0}^{\xi}=x\right\}$. Recall that $h_{\xi}(x)$ was defined in (42).

Computing $\tilde{h}_{\xi}(x)$ is also easiest in the two extreme cases. When $\xi=1, \tilde{K}^{1}$ on the negative integers is a symmetric, simple random walk, which means that the process cannot escape to negative infinity. Hence, $\tilde{h}_{1}(x)=1$. Similarly, $\tilde{h}_{-1}(x)=0$.

Now, we can handle the more interesting cases with $-1<\xi<1$. From the second equation in (32) with $\xi^{*}=1$,

$$
\tilde{F}_{\xi}(x, \ell)=\frac{h_{\xi}(\ell)}{h_{1}(\ell)} \frac{h_{1}(x)}{h_{\xi}(x)} \tilde{F}_{1}(x, \ell),
$$

and $\tilde{F}_{1}(x, \ell)=1$ whenever $\ell>x$. Since the walk is nearest neighbor and transient, letting $\ell \rightarrow \infty$ gives the desired escape probability

$$
\begin{aligned}
\tilde{h}_{\xi}(x) & =\frac{h_{1}(x)}{h_{\xi}(x)} \frac{1+\xi}{2} \\
& =\frac{1+|x|+x}{1+|x|+\xi x} \frac{1+\xi}{2}
\end{aligned}
$$


The analogous result with $\xi^{*}=-1$ and $\ell \rightarrow-\infty$ is that

$$
1-\tilde{h}_{\xi}(x)=\frac{h_{-1}(x)}{h_{\xi}(x)} \frac{1-\xi}{2}
$$

Combining the two gives the representation

$$
h_{\xi}(x)=\frac{1+\xi}{2} h_{1}(x)+\frac{1-\xi}{2} h_{-1}(x) \quad \text { for } \xi \in[-1,1] .
$$

Although we have determined the escape probabilities for all $h$-transforms, the escape probabilities when $h=\hat{h}$ defined in (9) will play a fundamental role in Yaglom limits in the $R$-transient case [11]. For our example, $\hat{h}=h_{0}$ as described in subsubsection 5.2.5; hence, the probability of escaping to positive infinity starting from state $x$ for this $h$-transform is

$$
\tilde{h}_{0}(x)=\frac{1+|x|+x}{2(1+|x|)}
$$

In particular, the probability measure $\pi_{x}$ describing the periodic Yaglom limit starting from $x$ can be represented as the following convex combination of two extremal measures

$$
\pi_{x}=\tilde{h}_{0}(x) \pi_{\infty}+\left(1-\tilde{h}_{0}(x)\right) \pi_{-\infty}
$$

Thus, the escape probabilities corresponding to $\hat{h}$ determine the proper weights.

5.2.11. Martin exit and entrance boundaries of the hub-and-two-spoke model. This section depends heavily on the works of Dynkin [8] and Woess [33, 34]. Among other things, the Martin exit boundary theory can characterize all positive harmonic functions, and the Martin entrance boundary theory, all invariant measures of an irreducible stochastic or substochastic matrix. Generally, there seems to be more interest in exit boundary theory since it is useful in describing the limiting behavior of transient processes; if left unspecified, Martin boundary theory usually refers to the exit boundary. Similarly, the $t$-Martin exit and entrance boundary theory can be used to describe all $t$-invariant harmonic functions and $t$-invariant measures. Again, there seems to be more interest in the exit boundary theory. Papers that study the $t$-Martin exit boundary of killed random walks include Ignatiouk-Robert [13, 14], Doney [6], Alili and Doney [1], Raschel [25], and Lecouvey and Raschel [20]. Maillard [21] identifies 
the $t$-invariant measures for the Bienaymé-Galton-Watson process $t \geq \rho$. The $\rho$ Martin entrance boundary for this process is trivial having a single point, and the corresponding $\rho$-invariant measure is the classic limit of Yaglom. When the $\rho$-Martin entrance boundary is trivial, it is impossible to have different (aperiodic or periodic) Yaglom limits starting from different initial states.

Fix $t \geq \rho$. Although $G_{(x, y)}(t)$ was defined in (4), the Martin boundary definitions will be slightly less ugly if we also define $G_{t}(x, y):=\sum_{n \geq 0}(1 / t)^{n} K^{n}(x, y)$. To construct the $t$-Martin boundaries, we define ${ }^{*} M$ and $M^{*}$, the $t$-Martin entrance and exit kernels respectively using 0 as the reference state, as:

$$
{ }^{*} M(x, y):=\frac{G_{t}(x, y)}{G_{t}(x, 0)} \text {, and } M^{*}(x, y):=\frac{G_{t}(x, y)}{G_{t}(0, y)} .
$$

Recall that $K$ is $R$-transient, and $1 / t \leq R$ so ${ }^{*} M$ and $M^{*}$ exist.

Let ${ }^{*} \bar{S}$ be the smallest compactification such that the $t$-Martin kernel ${ }^{*} M(x, y)$ extends continuously in $x$; i.e., $x_{\infty} \in{ }^{*} \bar{S}$ if there is a sequence $x_{n} \in S$ such that ${ }^{*} M\left(x_{n}, y\right)$ converges for every $y$. The limiting measure on $S$ is denoted by ${ }^{*} M\left(x_{\infty}, \cdot\right)$. Let $\partial^{*} \bar{S}:={ }^{*} \bar{S} \backslash S$ be the boundary of ${ }^{*} \bar{S} . \partial^{*} \bar{S}$ is called the $t$-Martin entrance boundary. For more details, see of [34, Chapter 7]

Similarly let $\bar{S}^{*}$ be the smallest compactification such that the $t$-Martin kernel $M^{*}(x, y)$ extends continuously in $y$; i.e., $y_{\infty} \in \bar{S}^{*}$ if there is a sequence $y_{n} \in S$ such that $M^{*}\left(x, y_{n}\right)$ converges for every $x$. The limiting function on $S$ is denoted by $M^{*}\left(\cdot, y_{\infty}\right)$. Let $\partial \bar{S}^{*}:=\bar{S}^{*} \backslash S$ be the boundary of $\bar{S}^{*} . \partial \bar{S}^{*}$ is called the $t$-Martin exit boundary.

We are particularly interested in the $\rho$-Martin entrance boundary. Though the $\rho$-Martin entrance boundary for substochastic matrices seems to have received little attention, it is ideally suited for studying Yaglom limits starting from a fixed state since the Yaglom limit (periodic or aperiodic) is a $\rho$-invariant QSD, and the $\rho$-Martin entrance boundary describes all $\rho$-invariant measures.

For the hub-and-two-spoke example, we will show that the $\rho$-Martin exit and entrance boundaries both have two points $\{-\infty,+\infty\}$. If $x_{n} \rightarrow-\infty$, then ${ }^{*} M\left(x_{n}, \cdot\right) \rightarrow$ $\sigma_{-1}(\cdot) / \sigma_{-1}(0)$; if $x_{n} \rightarrow+\infty$, then ${ }^{*} M\left(x_{n}, \cdot\right) \rightarrow \sigma_{1}(\cdot) / \sigma_{1}(0)$. Thus, we can extend ${ }^{*} M$ continuously to the boundary $\partial^{*} \bar{S}=\{-\infty,+\infty\}$ by defining ${ }^{*} M(-\infty, \cdot):=$ $\sigma_{-1}(\cdot) / \sigma_{-1}(0)$ and ${ }^{*} M(+\infty, \cdot):=\sigma_{1}(\cdot) / \sigma_{1}(0)$. Since the two limits differ, we cannot extend $M$ continuously with a smaller compactification. Similarly, we will show that 
if $y_{n} \rightarrow-\infty$, then $M^{*}\left(\cdot, y_{n}\right) \rightarrow h_{-1}(\cdot)=: M^{*}(\cdot,-\infty)$ and that if $y_{n} \rightarrow+\infty$, then $M^{*}\left(\cdot, y_{n}\right) \rightarrow h_{1}(\cdot)=: M^{*}(\cdot,+\infty)$, which shows that the $\rho$-Martin exit boundary is $\partial \bar{S}^{*}=\{-\infty,+\infty\}$.

To verify the claimed limits for the entrance boundary, let us derive a more convenient expressions for the $\rho$-Martin entrance kernel given in (57). From (57) with $t=\rho$ and using (33),

$$
\begin{aligned}
{ }^{*} M(x, y) & =\frac{\sigma_{\xi^{*}}(y)}{\sigma_{\xi^{*}}(0)} \frac{\overleftarrow{G}_{\xi^{*}}(y, x)}{\overleftarrow{G}_{\xi^{*}}(0, x)} \\
& =\frac{\sigma_{\xi^{*}}(y)}{\sigma_{\xi^{*}}(0)} \frac{\overleftarrow{F}_{\xi^{*}}(y, x)}{\overleftarrow{F}_{\xi^{*}}(0, x)} \overleftarrow{G}_{\xi^{*}}(x, x) \\
& =\frac{\sigma_{\xi^{*}}(y)}{\sigma_{\xi^{*}}(0)} \frac{\overleftarrow{F}_{\xi^{*}}(x, x)}{\overleftarrow{F}_{\xi^{*}}(0, x)}
\end{aligned}
$$

Now, it is simple to compute the claimed limits for ${ }^{*} M\left(x_{n}, y\right)$. If $x_{n} \rightarrow-\infty$, choose $\xi^{*}=-1$ so that ${ }^{*} M\left(x_{n}, y\right) \rightarrow \sigma_{-1}(y) / \sigma_{-1}(0)$. On the other hand, if $x_{n} \rightarrow+\infty$, choose $\xi^{*}=1$ so that ${ }^{*} M\left(x_{n}, y\right) \rightarrow \sigma_{1}(y) / \sigma_{1}(0)$.

Similarly, the $\rho$-Martin exit kernel can be rewritten using (34) to obtain

$$
M^{*}(x, y)=\frac{\tilde{F}_{\xi^{*}}(x, y)}{\tilde{F}_{\xi^{*}}(0, y)} \frac{h_{\xi^{*}}(x)}{h_{\xi^{*}}(0)}
$$

which makes it easy to verify the claimed limits for $M^{*}\left(x, y_{n}\right)$.

For the hub-and-two-spoke model, we have the representation of all positive $\rho$ invariant measures by (55) and all positive $\rho$-harmonic functions by (56). The mapping where state $x \in \mathbb{Z}$ is mapped to $x /(1+|x|)$ is a homeomorphism that connects the boundary points $-\infty$ to -1 and $+\infty$ with 1 . With this homeomorphism, (56) is the general integral representation of the $\rho$-harmonic functions over the $\rho$-Martin exit boundary (see Theorem 6 in [8]) and (55) will be the integral representation of the $\rho$-invariant measures over the $\rho$-Martin entrance boundary (see Theorem 11 in [8]).

From [28], we know that the hub-and-one-spoke model also has a unique quasistationary distribution for every $t \in(\rho, 1)$. We now argue that - similar to the situation when $t=\rho$ - the hub-and-two-spoke model has a family of quasi-stationary distributions for every $t \in(\rho, 1)$, which can be normalized to give a $t$-invariant quasi-stationary distribution. 
Fix $t \in(\rho, 1)$. Let $0<s_{1}<s_{2}<1$ be the roots of the $f(r)=a r^{2}-t r+b$, which must be real and distinct since the discriminant is positive. Define

$$
\sigma_{-}(x)= \begin{cases}\frac{s_{1}^{x}}{2} & \text { for } x \geq 1 \\ C s_{1}^{|x|}+\frac{s_{2}}{s_{2}-s_{1}} s_{2}^{|x|} & \text { for } x \leq-1 \\ 1 & \text { for } x=0\end{cases}
$$

where $C=\left(1 / 2-s_{2} /\left(s_{2}-s_{1}\right)\right)$. Even though $C<0, \sigma_{-}(x)$ is strictly positive since $\sigma_{-}(x) \geq s_{1}^{|x|} / 2$ for all $x$. In addition, $\sigma_{-}$is summable since $\sigma_{-}(x) \leq s_{2}^{|x|}$ for all $x$. Thus, $\sigma_{-}$could be normalized to be a proper probability distribution. Furthermore, $\sigma_{-}$is a $t$-invariant measure for $K$. For $x \geq 2$, the time reversal of $K$ with respect to $\sigma_{-}$ gives $\overleftarrow{K}(x, x-1)=b /\left(t s_{1}\right)$ and $\overleftarrow{K}(x, x+1)=a s_{1} / t$. For $x \geq 2$, the drift $b /\left(t s_{1}\right)-a s_{1} / t$ is negative if $s_{1}<\sqrt{b / a}$. But the smaller root $s_{1}$ is less than $\sqrt{b / a}$ since

$$
f(\sqrt{b / a})=(\rho-t) \sqrt{b / a}<0
$$

Since the time reversal is 1 -transient, the reversed process converges to $-\infty$. Thus, the $t$-Martin entrance boundary will have a point $-\infty$, and the $t$-Martin entrance kernel at $-\infty$ is $\sigma_{-}$, which is a minimal $t$-invariant measure. By symmetry, $\sigma_{+}(x):=\sigma_{-}(-x)$ is a different minimal $t$-invariant measure corresponding to a different $t$-Martin boundary point $+\infty$. Since a nearest neighbor random walk on the integers could have at most two points in the (full) Martin compactification and we have found two minimal $t$ invariant measures, we have found the whole Martin compactification for each $t>\rho$. Any convex combination of the two minimal $t$-invariant measures is also a $t$-invariant measure. Since the $t$-Martin exit boundary does not depend on $t$ for $t \in[\rho, 1)$ and satisfies the conditions given by Woess [33, page 301], the Martin entrance boundary is strictly stable. From reversibility, the dual $t$-harmonic functions can be determined using the reversibility measure $\gamma(43)$. The Martin exit boundary is also strictly stable.

\subsection{Time until returning to state zero}

We construct an example where the ratio limit $\hat{h}(\cdot)$ that is defined just before Prop. 6 is not $\rho$-harmonic; indeed, this example does not have any $\rho$-harmonic functions. This example does not contradict Prop. 6 since the support of $K(u, \cdot)$ will be infinite when $u=0$. Thus, the finite support assumption is needed in Prop. 6 and, in general, we 
cannot limit attention to $\rho$-harmonic functions when determining $\hat{h}(\cdot)$. Despite the above, we compute the limiting conditional distribution. In addition, if we consider $K^{2}$ with state space the even integers, we have an example with a Yaglom limit that falls outside of Kesten's sufficient conditions [18] since his condition (1.4u) does not hold.

This simple example has several other interesting aspects. The $\rho$-Martin exit kernel at $+\infty$ is the same as the $\rho$-Martin kernel at state 0 , which leads to a divergence of approaches on whether to include $+\infty$ in the boundary. Doob [7] and Kemeny, Snell and Knapp [17] would not include the point $+\infty$, while Hunt [12], Dynkin [8], and Woess [33, 34] would include $+\infty$ allowing $S$ to remain discrete in the induced topology; see the discussion at [34, page 189]. We follow the latter group.

The example is a variation on the hub-and-one-spoke example. Returns to state 0 in the hub-and-spoke model form a terminating renewal process. Define a sub-Markov chain where-prior to absorption-the state is the remaining lifetime of this renewal process; that is, the number of steps until being in state 0 .

The state space is $\mathbb{N}_{0}$, and the substochastic transition matrix is (recycling the notation $K$ )

$$
K=\left[\begin{array}{cccccc}
f_{1} & f_{2} & f_{3} & f_{4} & f_{5} & \cdots \\
1 & 0 & 0 & 0 & 0 & \cdots \\
0 & 1 & 0 & 0 & 0 & \cdots \\
\vdots & & & & &
\end{array}\right]
$$

where $f_{n}$ is the coefficient of $z^{n}$ in the generating function $F(z)$ given in the beginning of subsection 5.1. For this example, $0=f_{1}=f_{3}=f_{5}=\ldots, F(R)=1 / 2, F(1)=b$, and $K$ is periodic with period 2 .

If both the hub-and-one-spoke and the remaining lifetime examples start in state 0 , then we can couple the two processes so that the times of visits to state 0 are identical. It immediately follows that this example is also $R$-transient with $\rho=2 \sqrt{a b}$. In addition, an asymptotic expression for $\mathbb{P}_{0}\{\zeta>2 n\}$ is given by the r.h.s. of (44) with $x=0$. This gives an asymptotic expression for the denominator of $\hat{h}_{0}(0)$ in (21) where the subscript 0 denotes the class of even states and $x_{0}=0$ is the reference state.

An asymptotic expression for the numerator can be found by noticing that $\mathbb{P}_{2 x}\{\zeta>$ 
$2 n\}=\mathbb{P}_{0}\{\zeta>2 n-2 x\}$. Simplifying gives $\hat{h}_{0}(2 x)=R^{2 x}$. Consequently, from the definition of $\hat{h}(\cdot)$ immediately before Prop. 6 , we have $\hat{h}(x)=R^{x}$ for $x \in \mathbb{N}_{0}$. Even though we have computed $\hat{h}(\cdot)$, we now show that $\hat{h}(\cdot)$ is not $\rho$-harmonic by showing that $K$ does not have any $\rho$-harmonic functions.

Solving $K h(x)=\rho h(x)$ for $x \geq 1$ gives $h(x)=R^{x} h(0)$, which is looking like $\hat{h}(\cdot)$ above. However, the equation $K h(0)=\rho h(0)$ simplifies to $\rho F(R) h(0)=\rho h(0)$, but $F(R)=1 / 2$. Thus, there is no non-zero solution, and $K$ does not have any $\rho$-harmonic functions. The function $\hat{h}(x)=R^{x}$ is $\rho$-superharmonic.

We can compute the limiting conditional distribution for the remaining lifetime example as follows. First, since

$$
\frac{K^{n}(x, y)}{K^{n}(x, S)}=\frac{K^{n-x}(0, y)}{K^{n-x}(0, S)}
$$

any limiting conditional distribution would not depend on the initial state, so we can assume that the initial state is 0 without loss of generality. (This would also be true if we looked at a two-spoke version of the remaining lifetime example.) Consequently, should it exist, let $\pi^{k}$ to be the periodic Yaglom limit on $S_{k}$ where $S_{0}=2 \mathbb{N}_{0}$ and $S_{1}$ is the odd positive integers analogous to (12) or (13). Similarly, should it exist, let $\pi$ denote the corresponding $\rho$-invariant QSD.

From the coupling argument, we know that

$$
\begin{aligned}
\pi^{0}(0) & =\lim _{n \rightarrow \infty} \frac{K^{2 n}(0,0)}{K^{2 n}(0, S)} \\
& =\frac{1-\rho^{2}}{a}
\end{aligned}
$$

and if a periodic Yaglom limit exists, that

$$
\pi\left(S_{0}\right)=1 /(1+\rho), \pi\left(S_{1}\right)=\rho /(1+\rho) \text { and } \pi(0)=\frac{1-\rho}{a} .
$$

For $y \geq 1$,

$$
\begin{aligned}
K^{2 n}(0,2 y) & =K^{2 n+2 y}(0,0)-\sum_{k=1}^{y} K^{2 n+2 y-2 k}(0,0) f_{2 k} \\
& \sim \frac{1}{\sqrt{\pi}} \frac{(4 a b)^{n+y}}{n^{3 / 2}}\left[1-\sum_{k=1}^{n} f_{2 k} R^{2 k}\right] \\
\pi^{0}(2 y) & =\rho^{2 y} \frac{1-\rho^{2}}{a}\left[1-\sum_{k=1}^{y} f_{2 k} R^{2 k}\right]
\end{aligned}
$$


where we used the coupling to see that $K^{n}(0,0)=P^{n}(1,1)$, (36) with $x=y=1$, and (44) with $x=0$. Since $\sum_{y \geq 1} \rho^{2 y} \sum_{1 \leq k \leq y} f_{2 k} R^{2 k}=b /\left(1-\rho^{2}\right)$, it follows that $\pi^{0}$ is a proper probability measure on $S_{0}$. Thus, for every $x \in S_{0}$, the hypotheses of Prop. 3 hold; hence, for every $x \in S_{0}$, there is a $\rho$-invariant QSD. But $K$ has a unique $\rho$-invariant QSD, which is given by

$$
\pi(y)=\rho^{y} \frac{(1-\rho)}{a}\left[1-\sum_{k=1}^{y} f_{k} R^{k}\right] \quad \text { for } y \in \mathbb{N}_{0},
$$

(and is quite different from the analogous result for the hub-and-one-spoke model). Consequently, the $\rho$-invariant QSD $\pi$ must describe the (periodic) limiting conditional behavior starting from any state $x$.

5.3.1. Exit and entrance boundaries for the time remaining until returning to zero For the time until returning to zero example, we examine the $\rho$-Martin exit and entrance boundaries. Unlike the hub-and-two-spoke model, the $\rho$-Martin entrance and exit boundaries are not the same. In addition, the minimal $\rho$-Martin exit boundary and the $\rho$-Martin exit boundary are not equal.

To compute the $\rho$-Martin kernels, use (34) so that

$$
G_{\rho}(x, y)=\tilde{G}(x, y) \frac{\hat{h}(x)}{\hat{h}(y)}
$$

where $\hat{h}(x)=R^{x}$,

$$
\tilde{K}=\left[\begin{array}{cccccc}
\tilde{f}_{1} & \tilde{f}_{2} & \tilde{f}_{3} & \tilde{f}_{4} & \tilde{f}_{5} & \cdots \\
1 & 0 & 0 & 0 & 0 & \cdots \\
0 & 1 & 0 & 0 & 0 & \cdots \\
\vdots & & & & &
\end{array}\right]
$$

and $\tilde{f}_{k}=R f_{k} \hat{h}(k-1) / \hat{h}(0)=f_{k} R^{k}$. Notice that $\tilde{K}(0, S)=F(R)=1 / 2$. To compute $\tilde{G}(x, y)$, notice that $\tilde{G}(x, y)=\mathbb{1}\{x \geq y>0\}+\tilde{G}(0, y)$ where $\mathbb{1}\{x \geq y>0\}$ is 1 if $x \geq$ $y>0$ and zero, otherwise. To compute $\tilde{G}(0, y)$, also notice that the number of upward excursions has a geometric distribution with parameter $1 / 2$, and the expected number of excursions is 1 . Each excursion visits $y$ with probability $\tilde{F}_{y}:=\tilde{f}_{y+1}+\tilde{f}_{y+2}+\ldots$ Thus, $\tilde{G}(0, y)=\tilde{F}_{y}$. 
Now, the $\rho$-Martin exit kernel is

$$
\begin{aligned}
M^{*}(x, y) & :=\frac{G_{\rho}(x, y)}{G_{\rho}(0, y)} \\
& =\frac{\hat{h}(x) \tilde{G}(x, y)}{\hat{h}(0) \tilde{G}(0, y)} \\
& =R^{x} \frac{\mathbb{1}\{x \geq y>0\}+\tilde{G}(0, y)}{\tilde{G}(0, y)}
\end{aligned}
$$

Since $M^{*}\left(x, y_{n}\right) \rightarrow R^{x}$ as $y_{n} \rightarrow \infty$, there is a single point $+\infty$ in the $\rho$-Martin exit boundary - even though $M^{*}(x,+\infty)=M^{*}(x, 0)=R^{x}$, a point is still added in the compactification of the state space; see [34, page 189]. As a consequence, the minimal $\rho$ Martin exit boundary is the empty set since there are no minimal $\rho$-harmonic functions; see [34, page 207]. Thus, for this example, the $\rho$-Martin exit boundary and the minimal $\rho$-Martin exit boundary are not the same.

\subsection{Age example}

Instead of considering the remaining lifetime as in the previous example, consider the age where the age is the number of steps since being in zero prior to absorption. The state space is $\mathbb{N}_{0}$, and the substochastic transition matrix is (recycling the notation $K$ again)

$$
K=\left[\begin{array}{cccccc}
0 & b & 0 & 0 & 0 & \cdots \\
r_{2} & 0 & 1-r_{2} & 0 & 0 & \cdots \\
r_{3} & 0 & 0 & 1-r_{3} & 0 & \cdots \\
\vdots & & & & &
\end{array}\right]
$$

where $r_{j}=f_{j} / \bar{F}_{j}$ and $\bar{F}_{j}=f_{j}+f_{j+1}+\ldots$ The age example and the remaining lifetime example can be coupled so that they are in zero at the same points in time and have the same time of absorption if both start in state 0 . Thus, the age example is also $R$-transient with $\rho=2 \sqrt{a b}$. This example is also periodic with period 2 since $0=r_{1}=r_{3}=\cdots$

The age example seems nicer than the remaining lifetime example since the support of $K(u, \cdot)$ is finite for all states $u$. Nonetheless, $K$ does not possess a $\rho$-invariant QSD. From Prop. 3, it follows that (12) cannot hold. Thus, the age example does not have a (periodic or aperiodic) Yaglom limit. 


\subsection{Duality without assuming reversibility}

In section 3 , we described a duality between $t$-invariant measures and $t$-harmonic functions that relied on reversibility. We describe a generalization that does not rely on reversibility.

For this duality to hold, we need the following two conditions:

- If * $M\left(z_{n}, y\right)$ converges to a minimal $t$-invariant measure $\sigma$ for some sequence $z_{n}$, then $M^{*}\left(x, z_{n}\right)$ also converges to a minimal $t$-harmonic function $h$.

- If $M^{*}\left(x, z_{n}\right)$ converges to a minimal $t$-harmonic function $h$ for some sequence $z_{n}$, then ${ }^{*} M\left(z_{n}, y\right)$ also converges to a minimal $t$-invariant measure $\sigma$.

A $t$-harmonic function $h$ is minimal if $h(0)=1$ and $h \geq h_{1}$ where $h_{1}$ is also $t$ harmonic implies that $h_{1} / h$ must be a constant; a minimal $t$-invariant measure is defined analogously. The above two properties imply that $\mathcal{M}^{*}={ }^{*} \mathcal{M}$ where $\mathcal{M}^{*}$ is the minimal $t$-Martin exit boundary [33, page 263-264] and ${ }^{*} \mathcal{M}$ is the minimal $t$-Martin entrance boundary.

Let $\sigma$ be any $t$-invariant measure for $K$, and let $h$ be any $t$-harmonic function. The Poisson-Martin integral provides a unique representation of $h[33,(24.18)]$ and $\sigma$ :

$$
\begin{aligned}
& h(x)=\int_{\mathcal{M}^{*}} M^{*}(x, \cdot) d \nu^{h} \\
& \sigma(y)=\int_{{ }^{*} \mathcal{M}}{ }^{*} M(\cdot, y) d \nu^{\sigma} .
\end{aligned}
$$

When $\mathcal{M}^{*}={ }^{*} \mathcal{M}$, we can say that $h$ and $\sigma$ are duals of each other if $\nu^{h}=\nu^{\sigma}$ in their Poisson-Martin representations.

We show that this concept of duality includes the duality described in section 3 when $K$ is reversible. Let $\gamma$ be the reversibility measure of $K$. For the first part of the argument, assume that $h$ is a minimal $t$-harmonic function. Hence, there exists a sequence of states $z_{n}$ such that $M^{*}\left(x, z_{n}\right) \rightarrow M^{*}\left(x, z_{\infty}\right)=: h(x)$, and the unique Poisson-Martin representation has $\nu^{h}$ being a point mass. We need to show that ${ }^{*} \mathcal{M}\left(z_{n}, x\right) \rightarrow \sigma(x):=h(x) \gamma(x)$ and that $\sigma$ is minimal to know that $\sigma$ is the dual of $h$ under the generalized definition. 
Now

$$
\begin{aligned}
{ }^{*} M\left(z_{n}, x\right) & =\frac{G_{t}\left(z_{n}, x\right)}{G_{t}\left(z_{n}, 0\right)} \\
& =\frac{\gamma(x) G_{t}\left(x, z_{n}\right) / \gamma\left(z_{n}\right)}{\gamma(0) G_{t}\left(0, z_{n}\right) / \gamma\left(z_{n}\right)} \\
& =\frac{\gamma(x)}{\gamma(0)} M^{*}\left(x, z_{n}\right) \\
& \rightarrow \gamma(x) h(x)=\sigma(x) .
\end{aligned}
$$

In addition, $\sigma$ is minimal. To see this, assume that $\sigma \geq \sigma_{1}$, which is also $t$-invariant. Hence, $h \geq h_{1}$ where $h_{1}(x)=\sigma_{1}(x) / \gamma(x)$. Since $h_{1}$ is also $t$-harmonic, $h / h_{1}$ is a constant, but that means $\sigma / \sigma_{1}$ is a constant, which means $\sigma$ is minimal. Thus, $\sigma$ must be the dual of $h$ under the generalized definition.

The second part of the argument is analogous to the first part except for starting off with a minimal $t$-invariant measure $\sigma$ and showing that $h(x)=\sigma(x) / \gamma(x)$ is the dual of $\sigma$.

From the first two parts of the argument, it follows that $\mathcal{M}^{*}={ }^{*} \mathcal{M}$ and that if $z_{\infty} \in \mathcal{M}^{*}$ then $M^{*}\left(x, z_{\infty}\right)={ }^{*} M\left(z_{\infty}, x\right) / \gamma(x)$. From the Poisson-Martin integral representation, it now follows that that the generalized definition of duality reduces to $h(x)=\sigma(x) / \gamma(x)$ when $K$ is reversible.

\section{Literature}

Van Doorn and Pollett [29] survey the vast literature on, and the interconnections among, Yaglom limits, limiting conditional distributions, QSDs and $t$-invariant measures. We discuss only the most relevant papers, and we consider only irreducible chains. Many papers are set in continuous time, but most results for continuous time processes have an obvious analog in discrete time [29, Section 3.4].

In the impressive paper Seneta and Vere-Jones [27], the authors show that the Yaglom limit does not depend on the initial state in the $R$-positive case, but they allow for the possibility that a Yaglom limit might depend on the starting state in the $R$-transient case. Seneta and Vere-Jones analyze several examples including the killed simple random walk on the nonnegative integers that we exploited and the BienayméGalton-Watson process. 
That the Yaglom limit might depend on the starting state seems to have been overlooked for two reasons: first, in all of the analyzed examples with Yaglom limits starting from a fixed state, the limit did not depend on the starting state; second, it seems like there should be a coupling argument showing that the limit does not depend on the starting state.

The connection between the $\rho$-Martin entrance boundary and Yaglom limits for $\rho \neq 1$ seems to have been largely overlooked except for Maillard [21], who studies the minimal $t$-Martin entrance boundary for $t \geq \rho$ for the subcritical Bienaymé-GaltonWatson process. He finds that the $\rho$-Martin entrance boundary is trivial and that there is a unique $\rho$-invariant QSD, which is the classic Yaglom limit. Breyer [3] made the profound connection between the space-time Martin entrance boundary and Yaglom limits but failed to realize that convergence to the space-time boundary could fail as in Kesten's example [18] (see below). Lalley [19] identifies the space-time Martin boundary of a nearest neighbor random walk (stochastic transition matrix) on a class of homogeneous trees.

Seneta and Vere-Jones [27] mention but do not pursue the domain of attraction problem. Most of the domain of attraction work has been concerned with situations where there is a unique $t$-invariant distribution for each $t \in[\rho, 1)$, and the question is which initial distributions are in the domain of attraction of a particular $t$-invariant distribution; see van Doorn [28] and Villemonais [32]. This is slightly different than subsubsection 5.2.7 where we have many $\rho$-invariant distributions, and we determine which initial distributions with a finite support are in the domain of attraction of a given $\rho$-invariant QSD.

The most amazing example appears in H. Kesten's tour de force [18]. Certainly, Kesten was aware that the Yaglom limit could depend on the starting state, but he does not even bother to mention it. Instead, he focuses on constructing an example of a sub-Markov chain possessing most every nice property possible including having at least one $\rho$-invariant QSD, but fails to have a Yaglom limit! Our far more pedestrian example has a different Yaglom limit for every starting state. Kesten's example and ours are similar. The only difference is that Kesten allows the process to stay in each state $x$ with probability $r_{x}$. Once the process leaves a state, the transition probabilities are identical to our example. By carefully choosing the values of $r_{x}$, Kesten constructs 
an example where $\mathbb{P}\left\{X_{n}>0 \mid X_{0}=0, X_{n} \in S\right\}$ oscillates as $n$ increases and does not converge. Hence, the Yaglom limit fails. Kesten [18] also gives general conditions guaranteeing the existence of a Yaglom limit that does not depend on the starting state but this result depends on conditions ensuring the uniqueness of the $\rho$-invariant probability or equivalently the triviality of the $\rho$-Martin entrance boundary.

\section{Acknowledgements}

We would like to thank Phil Pollett for his helpful comments, Vadim Kaimanovich for fielding some Martin boundary questions, and Jaime San Martin for several technical suggestions. We would also like to thank the referees for their comments, suggestions and questions, which we believe substantially improved the paper.

\section{References}

[1] Alili, L. And Doney, R. A. (2001). Martin boundaries associated with a killed random walk. Ann. Inst. H. Poincaré Probab. Statist. 37, 313-338. 5.2.11

[2] Billingsley, P. (1995). Probability and Measure third ed. Wiley Series in Probability and Mathematical Statistics. John Wiley \& Sons Inc., New York, NY. A Wiley-Interscience Publication. 2

[3] Breyer, L. A. (1998). Quasistationarity and Martin boundaries: Conditioned processes. http://www.lbreyer.com/preprints.html. 6

[4] Clark, P. L. (2012). Honors calculus. http://math.uga.edu/ pete/2400full.pdf. 2

[5] Clark, P. L. (2012). Sequences and series: A sourcebook. http://math.uga.edu/ pete/3100supp.pdf. 2

[6] Doney, R. A. (1998). The Martin boundary and ratio limit theorems for killed random walks. J. London Math. Soc. (2) 58, 761-768. 5.2.11

[7] Doов, J. L. (1959). Discrete potential theory and boundaries. J. Math. Mech. 8, 433-458; erratum 993. 5.3 
[8] Dynkin, E. B. (1969). Boundary theory of Markov processes (the discrete case). Russian Mathematical Surveys 24, 1. 2.2, 5.2.11, 5.2.11, 5.3

[9] Ferrari, P. A. and Rolla, L. T. (2015). Yaglom limit via Holley inequality. Braz. J. Probab. Stat. 29, 413-426. 4

[10] Foley, R. D. and McDonald, D. R. Yaglom limits for $R$-recurrent chains. manuscript. 2

[11] Foley, R. D. And McDonald, D. R. Yaglom limits for $R$-transient chains and the space-time Martin boundary. manuscript. 5.2.10

[12] Hunt, G. A. (1960). Markoff chains and Martin boundaries. Illinois J. Math. 4, 313-340. 5.3

[13] Ignatiouk-Robert, I. (2008). Martin boundary of a killed random walk on a half-space. J. Theoret. Probab. 21, 35-68. 5.2.11

[14] Ignatiouk-Robert, I. And Loree, C. (2010). Martin boundary of a killed random walk on a quadrant. Ann. Probab. 38, 1106-1142. 5.2.11

[15] Jacka, S. D. And Roberts, G. O. (1995). Weak convergence of conditioned processes on a countable state space. J. Appl. Probab. 32, 902-916. 1, 2.4 .1

[16] Kelly, F. P. (1979). Reversibility and stochastic networks. John Wiley \& Sons, Ltd., Chichester. Wiley Series in Probability and Mathematical Statistics. 3

[17] Kemeny, J. G., Snell, J. L. And Knapp, A. W. (1976). Denumerable Markov chains second ed. Springer-Verlag, New York-Heidelberg-Berlin. With a chapter on Markov random fields, by David Griffeath, Graduate Texts in Mathematics, No. 40.5 .3

[18] Kesten, H. (1995). A ratio limit theorem for (sub) Markov chains on $\{1,2, \cdots\}$ with bounded jumps. Advances in applied probability 652-691. 2, 2.4.1, 2.4.1, 2.4.1, 5.3, 6

[19] Lalley, S. P. (1991). Saddle-point approximations and space-time Martin boundary for nearest-neighbor random walk on a homogeneous tree. J. Theoret. Probab. 4, 701-723. 6 
[20] Lecouvey, C. and Raschel, K. (2015). t-martin boundary of killed random walks in the quadrant. ArXiv e-prints. 5.2.11

[21] Maillard, P. (2015). The -invariant measures of subcritical Bienaym-GaltonWatson processes. ArXiv e-prints. 5.2.11, 6

[22] OdLyzko, A. M. (1995). Asymptotic enumeration methods. In Handbook of combinatorics. ed. R. L. Graham, M. Groetschel, and L. Lovasz. vol. 2. Elsevier pp. 1063-1229. http://www.dtc.umn.edu/ odlyzko/doc/asymptotic.enum.pdf. 5.2.1

[23] Pollett, P. K. (1988). Reversibility, invariance and $\mu$-invariance. Adv. in Appl. Probab. 20, 600-621. 3

[24] Pollett, P. K. (1989). The generalized Kolmogorov criterion. Stochastic Process. Appl. 33, 29-44. 3, 7

[25] Raschel, K. (2009). Random Walks in the Quarter Plane Absorbed at the Boundary : Exact and Asymptotic. ArXiv e-prints. 5.2.11

[26] Seneta, E. (2006). Non-negative matrices and Markov chains. Springer Science \& Business Media. 2.2

[27] Seneta, E. and Vere-Jones, D. (1966). On quasi-stationary distributions in discrete-time Markov chains with a denumerable infinity of states. Journal of Applied Probability 403-434. 1, 2.2, 2.4, 5, 5, 5.1, 5.2.2, 5.2.3, 6

[28] van Doorn, E. A. (1991). Quasi-stationary distributions and convergence to quasi-stationarity of birth-death processes. Adv. in Appl. Probab. 23, 683-700. $5.2 .11,6$

[29] van Doorn, E. A. And Pollett, P. K. (2013). Quasi-stationary distributions for discrete-state models. European journal of operational research 230, 1-14. 6

[30] van Doorn, E. A. And SchriJner, P. (1995). Geomatric ergodicity and quasistationarity in discrete-time birth-death processes. The Journal of the Australian Mathematical Society. Series B. Applied Mathematics 37, 121-144. 2.2 
[31] Vere-Jones, D. ET AL. (1967). Ergodic properties of nonnegative matrices i. Pacific J. Math 22, 361-386. 2.4, 2.5

[32] Villemonais, D. (2015). Minimal quasi-stationary distribution approximation for a birth and death process. Electron. J. Probab. 20, no. 30, 1-18. 6

[33] Woess, W. (2000). Random Walks on Infinite Graphs and Groups. Cambridge Tracts in Mathematics. Cambridge University Press. 5.2.11, 5.2.11, 5.3, 5.5

[34] Woess, W. (2009). Denumerable Markov chains. EMS Textbooks in Mathematics. European Mathematical Society (EMS), Zürich. Generating functions, boundary theory, random walks on trees. 5.2.11, 5.2.11, 5.3, 5.3.1 